TRANSACTIONS OF THE

AMERICAN MATHEMATICAL SOCIETY

Volume 350, Number 2, February 1998, Pages 501-522

S $0002-9947(98) 01730-9$

\title{
LÉVY PROCESSES IN SEMISIMPLE LIE GROUPS AND STABILITY OF STOCHASTIC FLOWS
}

\author{
MING LIAO
}

\begin{abstract}
We study the asymptotic stability of stochastic flows on compact spaces induced by Levy processes in semisimple Lie groups. It is shown that the Lyapunov exponents can be determined naturally in terms of root structure of the Lie group and there is an open subset whose complement has a positive codimension such that, after a random rotation, each of its connected components is shrunk to a single moving point exponentially under the flow.
\end{abstract}

\section{INTRODUCTION}

In this paper, we are concerned with the asymptotic stability of the stochastic flows induced by Lévy processes in noncompact semisimple Lie groups.

Although the class of such stochastic flows is rather narrow, it includes many interesting examples. For example, let $S^{n-1}$ be the unit sphere in $R^{n}$ and let $Y_{i}$ be the orthogonal projection to $S^{n-1}$ of the coordinate vector field $\partial / \partial x_{i}$ on $R^{n}$. Consider the following SDE (stochastic differential equation) on $S^{n-1}$ :

$$
d x_{t}=\sum_{i=1}^{n} c_{i} Y_{i}\left(x_{t}\right) \circ d w_{t}^{i}
$$

where the $c_{i}$ 's are arbitrary constants, $w_{t}=\left(w_{t}^{1}, \ldots, w_{t}^{n}\right)$ is an $n$-dimensional Wiener process and od denotes the Stratonovich differential. The solution flow of this SDE can be regarded as a Lévy process in the connected Lorentz group $S O_{+}(1, n)$. When all $c_{i}=1$, the stochastic flow is called the gradient flow and has been studied by Baxendale [2] and Elworthy [4].

The above example has the following physical interpretation. Imagine $S^{n-1}$ is the surface of a globe, fixed at its center, on which particles are distributed. Assume the particles can move freely along the surface. The above stochastic flow describes the motion of these particles under the influence of a random air flow. We may also assume that the globe is subject to random rotations, either continuously or at randomly spaced intervals, to obtain a stochastic flow induced by a more general Lévy process in $S O_{+}(1, n)$.

We will see that the asymptotic properties of the stochastic flows, at least qualitatively, are completely determined by the structure of the group in which they lie. As for the above example with $S O_{+}(1, n)$, it will be shown that almost surely, there is a random point in $S^{n-1}$ such that all the particles off this point will be swept to a single "moving" point exponentially fast by the stochastic flow.

Received by the editors June 19, 1995.

1991 Mathematics Subject Classification. Primary 58G32; Secondary 60H10.

Key words and phrases. Levy processes, semisimple Lie groups, stochastic flows. 
In $[9,10,11]$, we considered the stochastic flows induced by a special type of Lévy processes, i.e., the horizontal diffusions, in noncompact semisimple Lie groups. Using the limiting properties of horizontal diffusions found in Malliavin and Malliavin [12], we were able to prove some very explicit results on the asymptotic stability of the stochastic flows, which include the results on gradient flows on spheres as special cases. However, as the horizontal diffusions are rather special, the applications of these results were limited. For example, they do not cover stochastic flows generated by the SDE (1) when the constants $c_{i}$ are not equal.

In this paper, we will apply the results found in Guivarc'h and Raugi [5] on the limiting properties of random walks on noncompact semisimple Lie groups, which extend some earlier results by Furstenberg and others (see the references in [5]). By adapting these results for Lévy processes, we will be able to treat the asymptotic stability of stochastic flows much more general than those considered in $[9,10,11]$.

After a brief introduction to semisimple Lie groups in Section 2 and Lévy processes in Lie groups in Section 3, we will state a basic result on the limiting properties of Lévy processes in Section 4. The discrete time version of this result is given in [5]. Although the corresponding result for Lévy processes does not follow directly from this, it can be proved by essentially the same arguments of [5]. We also discuss some sufficient conditions for the desired limiting properties to hold.

In Section 5, we will obtain an integral formula for the rates at which the Lévy process tends to infinity. These rates determine the Lyapunov exponents of the stochastic flows to be discussed later. Our formula is more explicit than the one obtained in [5], and may lead to explicit calculation of the rates in some special cases.

Our main results on the asymptotic stability of the stochastic flows induced by Lévy processes are contained in Sections 6,7 and 8 . Let $G$ be a noncompact semisimple Lie group and let $Q$ be a closed subgroup. A Lévy process $g_{t}$ in $G$ can be naturally regarded as a stochastic flow on the left coset space $Q \backslash G$ via the right action of $G$. Although it is perhaps more customary to work with the left action, the use of the right action for stochastic flows is natural because the Lévy processes defined here are left invariant, which is consistent with most literature on this subject.

The local stability of the stochastic flow is determined by its Lyapunov exponents, which give the exponential rates at which the distance between near points grows or decays. We will see that all the Lyapunov exponents, together with the associated filtration of the tangent space, can be expressed using the roots of $G$. We will also obtain the global structure of stability, namely, we will show that there exist an open subset $\Lambda$ of $Q \backslash G$ whose complement has a positive codimension, and a random "rotation" $k_{\omega}$, such that almost surely, each connected component of $k_{\omega}(\Lambda)$ is shrunk to a single point exponentially fast under the flow. The set $\Lambda$ has a natural structure and, in concrete examples, can be determined explicitly. The random open set $k_{\omega}(\Lambda)$ is the stable manifold of the stochastic flow mentioned in [3]. It is worth noting that the set $\Lambda$ is completely determined by the group $G$ and is independent of the process $g_{t}$. Our results substantially extend the results obtained previously. Section 7 contains several technical lemmas used in proving the result for Lyapunov exponents. The approach is different from the one given in [9], which works only for horizontal diffusions.

In the last section, we will apply our theory to study several stochastic flows on spheres induced by Lévy processes in $S L(n, R)$ and $S O_{+}(1, n)$. 


\section{Some Preliminaries}

Throughout, we will assume that $G$ is a semisimple Lie group of noncompact type with a finite center. The reader is referred to [6] for the general theory of semisimple Lie groups. A typical example is given by $G=S L(n, R)$, the group of $n$ by $n$ real matrices of determinant one.

Let $K$ be a maximal compact subgroup of $G$. We will always use capital Roman letters for Lie groups and the corresponding script letters for their Lie algebras. Hence, $\mathcal{G}$ and $\mathcal{K}$ will be respectively the Lie algebras of $G$ and $K$.

Any $X \in \mathcal{G}$ is a tangent vector of $G$ at the identity element $e$ of $G$. For any $g \in G$, we will let $g X$ and $X g$ be respectively the vectors at $g$ obtained by left and right translations. For matrix groups, this notation coincides with the matrix multiplication.

The Killing form $B$ of $\mathcal{G}$ is defined by $B(X, Y)=\operatorname{Trace}[\operatorname{ad}(X) \operatorname{ad}(Y)]$, for $X, Y \in$ $\mathcal{G}$, where $\operatorname{ad}(X): \mathcal{G} \rightarrow \mathcal{G}$ is defined by $\operatorname{ad}(X) Y=[X, Y]$ (Lie bracket). The Lie group $G$ being semisimple means that $B$ is nondegenerate. Let $\mathcal{P}$ be the orthogonal complement of $\mathcal{K}$ in $\mathcal{G}$ with respect to $B$. It is $A d(K)$-invariant in the sense that $A d(k) Y \in \mathcal{P}$ for any $Y \in \mathcal{P}$ and $k \in K$, where $A d(k) Y=k Y k^{-1}$. The Killing form $B$ is positive definite when restricted to $\mathcal{P}$ and negative definite when restricted to $\mathcal{K}$. It induces an $A d(K)$-invariant inner product $\langle\cdot, \cdot\rangle$ on $\mathcal{G}$ by setting it equal to $B$ on $\mathcal{P},-B$ on $\mathcal{K}$ and keeping the orthogonality of $\mathcal{P}$ and $\mathcal{K}$.

Let $\mathcal{A}$ be a maximal abelian subspace of $\mathcal{P}$. A nonzero linear functional $\alpha$ on $\mathcal{A}$ is called a root if the vector space

$$
\mathcal{G}_{\alpha}=\{X \in \mathcal{G} ; \quad \operatorname{ad}(H) X=\alpha(H) X \text { for } H \in \mathcal{A}\}
$$

is nontrivial. The space $\mathcal{G}_{\alpha}$ is called the root space, any $X \in \mathcal{G}_{\alpha}$ a root vector, and $\operatorname{dim}\left(\mathcal{G}_{\alpha}\right)$ the multiplicity of $\alpha$. We have $\mathcal{G}=\mathcal{G}_{0} \oplus \sum_{\alpha} \mathcal{G}_{\alpha}$ as an orthogonal decomposition with respect to $\langle\cdot, \cdot\rangle$. Let $A$ be the connected abelian subgroup of $G$ with $\mathcal{A}$ as Lie algebra.

The hyperplanes $\alpha=0$ divide $\mathcal{A}$ into several open convex cones called Weyl chambers. Fix a Weyl chamber $\mathcal{A}_{+}$. A root $\alpha$ is said to be positive (relative to $\mathcal{A}_{+}$) if $\alpha>0$ on $\mathcal{A}_{+}$. If a root is not positive, then it is $-\alpha$ for some positive root $\alpha$ and will be called a negative root.

Since $\left[\mathcal{G}_{\alpha}, \mathcal{G}_{\beta}\right] \subset \mathcal{G}_{\alpha+\beta}\left(\mathcal{G}_{\alpha+\beta}=\{0\}\right.$ if $\alpha+\beta$ is not a root or zero), $\mathcal{N}=\sum_{\alpha>0} \mathcal{G}_{-\alpha}$ and $\mathcal{N}^{+}=\sum_{\alpha>0} \mathcal{G}_{\alpha}$ are both Lie subalgebras of $\mathcal{G}$, where the summations are taken over all positive roots. Let $N$ and $N^{+}$be respectively the connected Lie subgroups of $G$ with $\mathcal{N}$ and $\mathcal{N}^{+}$as Lie algebras. Both are nilpotent groups. We have the following two versions of Iwasawa decompositions:

$$
G=N A K=N^{+} A K
$$

in the sense that the maps $(n, a, k) \mapsto g=n a k$ and $\left(n^{\prime}, a, k\right) \mapsto g=n^{\prime} a k$ are, respectively, diffeomorphisms from $N \times A \times K$ and $N^{+} \times A \times K$ onto $G$.

Any $g \in G$ can be written as $g=x \exp \left(H^{+}\right) y$ for some $x, y \in K$ and a unique $H^{+} \in \overline{\mathcal{A}_{+}}$, the closure of $\mathcal{A}_{+}$. This is called the Cartan decomposition. Although the choice for $(x, y)$ is not unique, when $H^{+} \in \mathcal{A}_{+}$, all the possible choices are given by $\left(x m, m^{-1} y\right)$ for $m \in M$, where $M$ is the centralizer of $A$ in $K$, i.e., $M=\{k \in K$; $k a k^{-1}=a$ for $\left.a \in A\right\}$.

For $G=S L(n, R), \mathcal{G}$ is $\operatorname{sl}(n, R)$, the space of $n$ by $n$ real matrices of trace zero with $[X, Y]=X Y-Y X, B(X, Y)=2 n \operatorname{Trace}(X Y)$ and $\langle X, Y\rangle=2 n \operatorname{Trace}\left(X Y^{*}\right)$, where $Y^{*}$ is the transpose of $Y$. One may take $K$ to be $S O(n)$, the subgroup 
of orthogonal matrices, whose Lie algebra $\mathcal{K}$ is the space $o(n)$ of skew-symmetric matrices. Then $\mathcal{P}$ is the space of symmetric matrices of trace zero. One may take $\mathcal{A}=\left\{\operatorname{diag}\left(a_{1}, a_{2}, \cdots, a_{n}\right) ; \sum_{i} a_{i}=0\right\}$ and $\mathcal{A}_{+}=\left\{\operatorname{diag}\left(a_{1}, a_{2}, \cdots, a_{n}\right) \in \mathcal{A}\right.$; $\left.a_{1}>a_{2}>\cdots>a_{n}\right\}$. The roots are $\alpha_{i j}$, defined by $\alpha_{i j}(H)=a_{i}-a_{j}$ for $H=\operatorname{diag}\left\{a_{1}, a_{2}, \ldots, a_{n}\right\}$. The corresponding root space is 1-dimensional and is spanned by $E_{i j}$, the matrix which has 1 at place $(i, j)$ and 0 elsewhere. The positive roots are given by $\alpha_{i j}$ with $i<j$. We have $A=\left\{\operatorname{diag}\left(a_{1}, a_{2}, \cdots, a_{n}\right) ; a_{i}>0\right.$ and $\left.\prod_{i} a_{i}=1\right\} ; M$ is the group of the diagonal matrices with \pm 1 as diagonal entries and with an even number of -1 's; and $N$ and $N^{+}$are, respectively, the groups of lower triangular matrices and upper triangular matrices with diagonal entries all equal to one.

Let $B=G /\left(N^{+} A M\right)$ and let $\pi: G \rightarrow B$ be the natural projection. Via the Iwasawa decomposition $G=N^{+} A K, B$ can be naturally identified with $K / M$. We note that $B$ is a left $G$-space in the sense that any $g \in G$ acts on $B$ on the left by sending $g^{\prime}\left(N^{+} A M\right)$ into $g g^{\prime}\left(N^{+} A M\right)$. Similarly, let $\tilde{B}$ be the left coset space $(N A M) \backslash G$ and let $\tilde{\pi}: G \rightarrow \tilde{B}$ be the natural projection. Via the Iwasawa decomposition $G=N A K, \tilde{B}$ can be identified with $M \backslash K$. We note that $\tilde{B}$ is a right $G$-space in the sense that any $g \in G$ acts on $\tilde{B}$ on the right by sending $(N A M) g^{\prime}$ into $(N A M) g^{\prime} g$.

Besides the Iwasawa and Cartan decompositions, there is a third very useful decomposition on $G$, called the Bruhat decomposition. Let $M^{\prime}$ be the normalizer of $A$ in $K$, i.e., $M^{\prime}=\left\{k \in K ; k A k^{-1} \subset A\right\}$. Then $M$ is a normal subgoup of $M^{\prime}$, and $W=M^{\prime} / M$ is a finite group called the Weyl group. Any $w \in W$ can be considered as a linear transformation on $\mathcal{A}$, defined by $w(H)=A d\left(m_{w}\right) H$, for $H \in \mathcal{A}$, where $m_{w} \in M^{\prime}$ represents $w \in W=M^{\prime} / M$. We have the following Bruhat decomposition:

$$
G=\bigcup_{w \in W} N m_{w} N^{+} A M=\bigcup_{w \in W} N A M m_{w} N^{+} \quad \text { (disjoint unions), }
$$

where both $N N^{+} A M$ and $N A M N^{+}$are open subsets of $G$ whose complements have positive codimensions. It follows that $\pi(N)$ and $\tilde{\pi}\left(N^{+}\right)$are, respectively, connected open subsets of $B$ and $\tilde{B}$ with positive-codimensional complements.

\section{LÉVy Processes}

A Lévy process $g_{t}$ in $G$ is a Markov process whose transition semigroup $P_{t}$ is given by $P_{t} f(g)=\int_{G} f(g \sigma) \mu_{t}(d \sigma)$, where $\left\{\mu_{t}\right\}$ is a family of probability measures on $G$ satisfying $\mu_{t} * \mu_{s}=\mu_{t+s}$ and $\mu_{t}$ converges weakly to $\delta_{e}$, the point mass at the identity element $e$ of $G$, as $t \rightarrow 0$. The convolution $\mu_{t} * \mu_{s}$ is defined by $\mu_{t} * \mu_{s}(\Gamma)=\int_{a b \in \Gamma} \mu_{t}(d a) \mu_{s}(d b)$. By taking a proper version, we may assume that $g_{t}$ has right continuous paths with left limits.

Our Lévy process is left invariant in the sense that for any $g \in G, g g_{t}$ is the same Markov process starting at $g g_{0}$. A right invariant Lévy process is obtained with semigroup $P_{t} f(g)=\int_{G} f(\sigma g) \mu_{t}(d \sigma)$.

Let $X_{1}, X_{2}, \ldots, X_{m}$ be a basis of $\mathcal{G}$ which is orthonormal with respect to the inner product $\langle\cdot, \cdot\rangle$ introduced in Section 2 . Any $X \in \mathcal{G}$ can be identified with the left invariant vector field $g X$, for $g \in G$. There are smooth functions $x_{1}, x_{2}, \ldots, x_{m}$ on $G$ such that $x_{i}(e)=0, \lim _{g \rightarrow \infty} x_{i}(g)$ exist and $X_{i} x_{j}(e)=\delta_{i j}$. In a neighborhood of $e, x_{i}$ can be defined by $g=\exp \left(\sum_{i} x_{i}(g) X_{i}\right)$. By [7], a Lévy process $g_{t}$ in $G$ can 
be characterized as a Markov process with generator given by

$$
\begin{aligned}
L f(g)= & \frac{1}{2} \sum_{i, j=1}^{m} a_{i j} X_{i} X_{j} f(g)+\sum_{i=1}^{m} b_{i} X_{i} f(g) \\
& +\int_{G}\left[f(g \tau)-f(g)-\sum_{i=1}^{m} x_{i}(\tau) X_{i} f(g)\right] \eta(d \tau),
\end{aligned}
$$

for any smooth function $f$ on $G$ with compact support, where $a_{i j}$ and $b_{i}$ are constants with $a_{i j}$ forming a symmetric nonnegative definite matrix, and $\eta$ is a measure on $G$ satisfying $\eta(\{e\})=0, \int_{U} \sum_{i=1}^{m} x_{i}^{2} d \eta<\infty$ and $\eta(G-U)<\infty$ for some compact neighborhood $U$ of $e$. We note that $a_{i j}$ and $\eta$ are independent of, but $b_{i}$ may depend on, the choice of $x_{i}$. The measure $\eta$ is called the Lévy measure of $g_{t}$. The process $g_{t}$ is continuous if and only if $\eta=0$.

Let $g_{t}=n_{t} a_{t} k_{t}$ be the Iwasawa decomposition $G=N A K$. Then $k_{t}$ is a Markov process in $K$. To see this, note that if $V$ is a right $G$-space, then $v g_{t}$ is a Markov process in $V$ for any $v \in V$, due to the left invariance of $g_{t}$. Applying this observation to the right $G$-space $(N A) \backslash G \equiv K$, we see that $k_{t}=(N A) g_{t}$ is a Markov process.

If the Lévy measure $\eta$ is finite, then the generator (4) can be written as

$$
\begin{aligned}
L f(g) & =\frac{1}{2} \sum_{i, j=1}^{m} a_{i j} X_{i} X_{j} f(g)+\sum_{i=1}^{m} b_{i} X_{i} f(g)+\int_{G}[f(g \sigma)-f(g)] \eta(d \sigma) \\
& =(1 / 2) \sum_{i=1}^{n} U_{i} U_{i} f(g)+U_{0} f(g)+\int_{G}[f(g \sigma)-f(g)] \eta(d \sigma),
\end{aligned}
$$

where $U_{1}, U_{2}, \ldots, U_{n} \in \mathcal{G}$ are chosen to satisfy $\sum_{i=1}^{n} U_{i} U_{i}=\sum_{i, j=1}^{m} a_{i j} X_{i} X_{j}$ and $U_{0}=\sum_{i=1}^{m} b_{i} X_{i}$. We note that $n$ is not necessarily equal to $m$, and the $b_{i}$ here are different from those in (4).

Applebaum and Kunita [1] obtained the stochastic integral equations satisfied by a general Lévy process. If the Lévy measure $\eta$ is finite, the equation takes the following simpler form:

$$
\begin{aligned}
f\left(g_{t}\right)= & f\left(g_{0}\right)+\int_{0}^{t} \sum_{i=1}^{n} U_{i} f\left(g_{s-}\right) \circ d w_{s}^{i}+\int_{0}^{t} U_{0} f\left(g_{s}\right) d s \\
& +\int_{0}^{t}\left[f\left(g_{s-} \sigma\right)-f\left(g_{s-}\right)\right] N(d s d \sigma),
\end{aligned}
$$

for any smooth function $f$ on $G$ with compact support, where $w_{t}=\left(w_{t}^{1}, \ldots, w_{t}^{n}\right)$ is an $n$-dimensional Wiener process and $N(d t d \sigma)$ is a Poisson point process on $[0, \infty) \times G$ with intensity measure given by $d t \eta(d \sigma)$. See, e.g., Section I.9 in [8] for the definition of Poisson point processes. We have, for any nonnegative measurable function $f(t, g)$ on $[0, \infty) \times G$,

$$
E\left[\int_{0}^{t} \int_{G} f(s, g) N(d s d g)\right]=\int_{0}^{t} \int_{G} f(s, g) \eta(d g) d s .
$$

Given $U_{0}, U_{1}, \ldots, U_{n} \in \mathcal{G}$ and a finite measure $\eta$ on $G$, the Lévy process $g_{t}$ given by (6) can be constructed explicitly as follows. Let $w_{t}=\left(w_{t}^{1}, \ldots, w_{t}^{n}\right)$ be an $n$ dimensional Wiener process, let $S_{i}$ be a sequence of exponential random variables with a common mean $1 / \eta(G)$, and let $\sigma_{1}, \sigma_{2}, \ldots$ be a sequence of $G$-valued random 
variables having the common distribution $\eta / \eta(G)$. Assume all the above objects are independent. Let $T_{i}=\sum_{j=1}^{i} S_{j}$ and $T_{0}=0$. Then $g_{t}$ can be obtained by solving the SDE

$$
d g_{t}=\sum_{i=1}^{n} g_{t} U_{i} \circ d w_{t}^{i}+g_{t} U_{0} d t
$$

for $T_{i}<t<T_{i+1}$ and letting the process jump at $t=T_{i}$ according to $g_{t}=g_{t-} \sigma_{i}$.

For $g \in G$, let $g=g_{N}^{I} g_{A}^{I} g_{K}^{I}$ be the Iwasawa decomposition $G=N A K$. At the Lie algebra level, for $X \in \mathcal{G}$, let $X=X_{\mathcal{N}}^{I}+X_{\mathcal{A}}^{I}+X_{\mathcal{K}}^{I}$ be the decomposition $\mathcal{G}=\mathcal{N} \oplus \mathcal{A} \oplus \mathcal{K}$. Here, the superscript $I$ indicates that the decomposition is taken with respect to the Iwasawa decomposition.

Let $g=n a k$ be the Iwasawa decomposition. Then

$$
g \sigma=\left(n a\left[k \sigma k^{-1}\right]_{N}^{I} a^{-1}\right)\left(a\left[k \sigma k^{-1}\right]_{A}^{I}\right)\left(\left[k \sigma k^{-1}\right]_{K}^{I} k\right) .
$$

Hence, $[g \sigma]_{A}^{I}=a\left[k \sigma k^{-1}\right]_{A}^{I}$ and $[g \sigma]_{K}^{I}=\left[k \sigma k^{-1}\right]_{K}^{I} k$. For $U \in \mathcal{G}$ and small $s>0$,

$$
\left[g e^{s U}\right]_{A}^{I} \approx a \exp \left(s[\operatorname{Ad}(k) U]_{\mathcal{A}}^{I}\right) \text { and }\left[g e^{s U}\right]_{K}^{I} \approx \exp \left(s[\operatorname{Ad}(k) U]_{\mathcal{K}}^{I}\right) k .
$$

Hence, if a smooth function $f(g)$ on $G$ depends only on the $A$-component $a$ of $g$, then

$$
U f(g)=\left.(d / d s) f\left(g e^{s U}\right)\right|_{s=0}=[A d(k) U]_{\mathcal{A}}^{I} f(a)
$$

and

$$
U U f(g)=\left[[A d(k) U]_{\mathcal{K}}^{I}, A d(k) U\right]_{\mathcal{A}}^{I} f(a)+[A d(k) U]_{\mathcal{A}}^{I}[\operatorname{Ad}(k) U]_{\mathcal{A}}^{I} f(a) .
$$

Let $a_{t}=\exp \left(H_{t}\right)$. For $a \in A$ with $a=e^{H}$, define $\log a=H$. Applying (6) with $f(g)=\log \left(g_{A}^{I}\right)=H$ and noting

$$
U_{i} f\left(g_{s-}\right) \circ d w_{s}^{i}=U_{i} f\left(g_{s-}\right) d w_{s}^{i}+(1 / 2) U_{i} U_{i} f\left(g_{s}\right) d s,
$$

we obtain

$$
\begin{aligned}
H_{t}=H_{0} & +\int_{0}^{t} \sum_{i=1}^{n}\left[\operatorname{Ad}\left(k_{s-}\right) U_{i}\right]_{\mathcal{A}}^{I} d w_{s}^{i} \\
& +\int_{0}^{t}\left\{\frac{1}{2} \sum_{i=1}^{n}\left[\left[\operatorname{Ad}\left(k_{s}\right) U_{i}\right]_{\mathcal{K}}^{I}, A d\left(k_{s}\right) U_{i}\right]_{\mathcal{A}}^{I}+\left[\operatorname{Ad}\left(k_{s}\right) U_{0}\right]_{\mathcal{A}}^{I}\right\} d s \\
& +\int_{0}^{t} \int_{G} \log \left[k_{s-} \sigma k_{s-}^{-1}\right]_{A}^{I} N(d s d \sigma) .
\end{aligned}
$$

\section{Basic Limiting Properties of Lévy Processes}

In this section, we will assume that $g_{t}$ is a Lévy process in $G$ with $g_{0}=e$ and $\mu_{t}$ is the distribution of $g_{t}$ on $G$.

For probability measures $\mu$ on $G$ and $\nu$ on a left $G$-space $V$, the convolution $\mu * \nu$ is the probability measure on $V$ defined by $\mu * \nu(\Gamma)=\int_{g v \in \Gamma} \mu(d g) \nu(d v)$. We will say that $\nu$ is $\mu$-invariant if $\nu=\mu * \nu$. Similarly, if $V$ is a right $G$-space, we will define $\nu * \mu$ by $\nu * \mu(\Gamma)=\int_{v g \in \Gamma} \nu(d v) \mu(d g)$ and will say that $\nu$ is $\mu$-invariant if $\nu=\nu * \mu$.

The measure $\nu$ on $V$ will be called a stationary measure (of $g_{t}$ ) if it is $\mu_{t^{-}}$ invariant for all $t>0$. If $V$ is a right $G$-space, $v g_{t}$ is a Markov process in $V$ starting from $v \in V$. In this case, a stationary measure $\nu$ on $V$ can be characterized as a 
probability measure such that the Markov process becomes a stationary process if started with $\nu$ as initial distribution.

Recall that $\pi: G \rightarrow B=G /\left(N^{+} A M\right)$ is the natural projection, and $\pi(N)$ is an open subset of $B$ whose complement has a positive codimension.

As in [5], a probability measure $\nu$ on $B$ will be called irreducible if it does not charge $g[\pi(N)]^{c}$, for any $g \in G$, where the superscript $c$ denotes the complement in $B$. It is clear that if $\nu$ does not charge any submanifold of $B$ of a positive codimension, then it is irreducible.

Let $G_{\mu}$ be the smallest closed subgroup of $G$ containing all $\operatorname{supp}\left(\mu_{t}\right), t>0$, where $\operatorname{supp}\left(\mu_{t}\right)$ is the support of $\mu_{t}$. We note that $G_{\mu}$ is also the smallest closed subgroup of $G$ containing the support of $\mu=\int_{0}^{\infty} e^{-t} d t \mu_{t}$. We will use $T_{\mu}$ to denote $\operatorname{supp}(\mu)$. Because the $\mu_{t}$ form a convolution semigroup, we see that $T_{\mu}$ is the smallest closed semigroup containing all $\operatorname{supp}\left(\mu_{t}\right)$.

A closed subgroup $G^{\prime}$ of $G$ will be called totally irreducible if $G^{\prime}$ is not contained in $\bigcup_{i=1}^{r} g_{i}\left(N N^{+} A M\right)^{c} x$ for some $x, g_{1}, \ldots, g_{r} \in G$. We note that if $G^{\prime}$ is not contained in a finite union of positive codimensional submanifolds of $G$, then $G^{\prime}$ is totally irreducible. By Lemme 2.12 in [5], if $G_{\mu}$ is totally irreducible, then any stationary measure on $B$ or $\tilde{B}$ is irreducible.

A sequence $g_{j} \in G$ will be called a contracting sequence if under the Cartan decomposition $g_{j}=x_{j} \exp \left(H_{j}^{+}\right) y_{j}, \alpha\left(H_{j}^{+}\right) \rightarrow \infty$ for any positive root $\alpha$.

Let $g \in G$ and let $\lambda$ be a probability measure on $B$. We define $g \lambda$ to be the probability measure given by $g \lambda(f)=\int f(g b) \lambda(d b)$ for $f \in C(B)$ (the space of continuous functions on $B$ ).

Theorem 1. Let $g_{t}$ be a Lévy process in $G$ with $g_{0}=e$ and let $G_{\mu}$ and $T_{\mu}$ be defined above. Assume $G_{\mu}$ is totally irreducible and $T_{\mu}$ contains a contracting sequence. Then for any irreducible distribution $\lambda$ on $B, g_{t} \lambda$ converges weakly to a point mass $\delta_{z}$ on $B$ as $t \rightarrow \infty$, where $z$ is a $B$-valued random variable.

Consequently, under the Cartan decomposition $g_{t}=x_{t} \exp \left(H_{t}^{+}\right) y_{t}$, almost surely, as $t \rightarrow \infty, \alpha\left(H_{t}^{+}\right) \rightarrow \infty$ for any positive root $\alpha$ and $\pi\left(x_{t}\right) \rightarrow z$. Moreover, the distribution of $z$ is the unique stationary measure on $B$.

Proof. As the proof of this theorem is similar to the proof of Théorème 2.6 in [5], we will only outline the main steps.

Let $(\Omega, P)$ be the underlying probability space. First assume that $\lambda$ is a stationary measure on $B$. Then it is irreducible. As an easy consequence of the stationarity of $\lambda$, one can show that, for any $f \in C(B), M_{t}=g_{t} \lambda(f)=\int f\left(g_{t} b\right) \lambda(d b)$ is a bounded martingale. It follows that for $P$-almost all $\omega \in \Omega, g_{t} \lambda$ converges weakly to some measure $\zeta(\omega)$ on $B$ as $t \rightarrow \infty$ (see the first part of Lemme 2.13 in [5]).

The second step is to show that $\zeta=\delta_{z}$ for some random point $z$ in $B$. For random walks, this is done by first showing that for $P \times \mu$-almost all $(\omega, \xi), g_{n}(\omega) \xi \lambda \rightarrow \zeta(\omega)$ weakly (the second part of Lemme 2.13), and then using this and the existence of a contracting sequence in $T_{\mu}$ to show that $\zeta$ is a point mass (Lemme 2.14). There is no need to prove this for Lévy processes, as the discrete time result has already established the desired conclusion, i.e., $\zeta$ is a point mass $\delta_{z}$.

To prove $\alpha\left(H_{t}^{+}\right) \rightarrow \infty$ for $\alpha>0$, it suffices to show that for almost all $\omega$, if $g_{j}$ is a sequence in $G$ such that $g_{j} \lambda \rightarrow \delta_{z(\omega)}$ weakly, then $g_{j}$ is contracting. This is a consequence of Lemme 2.11 in [5]. The convergence $\pi\left(x_{t}\right) \rightarrow z$ follows from the observation that any limiting point of $\pi\left(x_{t}\right)$ must be the point $z$ above. This shows that the random point $z$ is independent of the choice of the stationary measure $\lambda$ 
on $B$. By the stationarity of $\lambda, \lambda=E\left[g_{t} \lambda\right] \rightarrow E\left[\delta_{z}\right]$. We see that $\lambda$ must be equal to the distribution of $z$. This proves the uniqueness of the stationary measure on $B$. Now we can see that $x_{t} \exp \left(H_{t}^{+}\right) y_{t} \lambda$ converges weakly to $\delta_{z}$ for any irreducible distribution $\lambda$ on $B$.

Remark 1. Recall that in the Cartan decomposition $g_{t}=x_{t} \exp \left(H_{t}^{+}\right) y_{t}$, the choice for $x_{t}$ and $y_{t}$ is not unique. By a suitable choice, we may assume that almost surely, $x_{t}$ converges to some $x_{\infty}$ in $K$.

The following purely geometric lemma is taken from [5] (see Corollaire (2.4)).

Lemma 1. If $g_{j}$ is a sequence in $G$ with Cartan decomposition $g_{j}=x_{j} \exp \left(H_{j}^{+}\right) y_{j}$ and Iwasawa decomposition $g_{j}=n_{j} \exp \left(H_{j}\right) k_{j}$, then the following two statements are equivalent.

(i) $x_{j} \rightarrow x \in N N^{+} A M$, and $\alpha\left(H_{j}^{+}\right) \rightarrow \infty$ for $\alpha>0$.

(ii) $n_{j} \rightarrow n$ in $N$, and $\alpha\left(H_{j}\right) \rightarrow \infty$ for $\alpha>0$.

Moreover, the above implies that $\pi(x)=\pi(n)$ and $H_{j}^{+}-H_{j}$ converges in $\mathcal{A}$.

The limiting properties of $g_{t}$ under the decomposition $G=N A K$ can be read off immediately from Theorem 1 and Lemma 1.

Corollary 1. Let $g_{t}=n_{t} \exp \left(H_{t}\right) k_{t}$ be the Iwasawa decomposition. Assume the hypotheses of Theorem 1. Then almost surely, as $t \rightarrow \infty, \alpha\left(H_{t}\right) \rightarrow \infty$ for $\alpha>0$ and $n_{t} \rightarrow n_{\infty}$ in $N$ with $\pi\left(n_{\infty}\right)=z$.

Now we give some sufficient conditions for the total irreducibility of $G_{\mu}$ and the existence of a contracting sequence in $T_{\mu}$ required in Theorem 1 . The conditions are given in terms of the generator $L$, so are easy to verify. Recall that $L$ is defined by (4).

As in Section 3, choose $U_{1}, U_{2}, \ldots, U_{n}$ such that $\sum_{i, j=1}^{m} a_{i j} X_{i} X_{j}=\sum_{i=1}^{n} U_{i} U_{i}$. Although such a choice is not unique, the space $\mathcal{L}$ spanned by $U_{1}, U_{2}, \ldots, U_{n}$ is independent of the choice. Let $V_{1}, V_{2}, \ldots, V_{m}$ be an orthonormal basis of $\mathcal{G}$ formed by the eigenvectors of the symmetric matrix $\left\{a_{i j}\right\}$ associated to the eigenvalues $\lambda_{1}, \lambda_{2}, \ldots, \lambda_{m}$. Let these eigenvalues be listed so that $\lambda_{i}>0$ for $i \leq k$ and $\lambda_{i}=0$ for $i>k$. We have $\sum_{i, j=1}^{m} a_{i j} X_{i} X_{j}=\sum_{i=1}^{k} \lambda_{i} V_{i} V_{i}$. The space $\mathcal{L}$ is spanned by $V_{1}, \ldots, V_{k}$. We note that if $U_{1}, \ldots, U_{n}$ are linearly independent, then $k=n$.

Let $G_{L}$ be the closed subgroup of $G$ generated by $\operatorname{supp}(\eta)$ and $\mathcal{L}$, i.e., $G_{L}$ is the smallest closed subgroup of $G$ containing $\operatorname{supp}(\eta)$ and all $e^{t U}$ for $U \in \mathcal{L}$ and real $t$.

Proposition 1. $G_{L}$ is contained in $G_{\mu}$. Consequently, if $G_{L}=G$, then $G_{\mu}=G$.

Proof. Let $P_{t}$ be the semigroup of $g_{t}$. For any smooth function $f$ on $G$ with compact support which vanishes near $e$, we have $\left.(d / d t) P_{t} f(e)\right|_{t=0}=L f(e)=\int_{G} f d \eta$. This shows that $\operatorname{supp}(\eta) \subset T_{\mu} \subset G_{\mu}$.

We now show that $\mathcal{L} \subset \mathcal{G}_{\mu}$, the Lie algebra of $G_{\mu}$. Fix an arbitrary vector $U$ in $\mathcal{G}$ orthogonal to $\mathcal{G}_{\mu}$. It suffices to show that $\left\langle U, V_{i}\right\rangle=0$ for $i \leq k$.

We have $V_{i}=\sum_{j} c_{i j} X_{j}$ and $\sum_{h} c_{i h} c_{j h}=\delta_{i j}$. We may choose $X_{1}, X_{2}, \ldots, X_{n}$ such that $X_{1}, \ldots, X_{r}(r \leq n) \operatorname{span} \mathcal{G}_{\mu}$. Since $U$ is orthogonal to $\mathcal{G}_{\mu}, U=$ $\sum_{j>r} c_{j} X_{j}$. Let $f$ be a nonnegative smooth function on $G$ which is equal to $\left(\sum_{j>r} c_{j} x_{j}\right)^{2}$ near $e$. By the properties of $x_{i}$ (see Section 3), we have $f(e)=0$, $V_{i} f(e)=0$ and $V_{i} V_{i} f(e)=2\left(\sum_{j>r} c_{j} c_{i j}\right)^{2}=2\left\langle U, V_{i}\right\rangle^{2}$. Hence,

$$
\left.(d / d t) P_{t} f(e)\right|_{t=0}=L f(e)=\sum_{i} \lambda_{i}\left\langle U, V_{i}\right\rangle^{2}+\int_{G} f d \eta .
$$


We may choose $x_{j}$ to vanish on $\exp \left(\mathcal{G}_{\mu}\right)$ for $j>r$, so we may also choose $f$ to vanish on $\exp \left(\mathcal{G}_{\mu}\right)$. Since $G_{\mu}$ is closed, it is a topological subgroup of $G$. We may modify the value of $f$ so that it vanishes outside a small neighborhood of $e$, hence, vanishes on $G_{\mu}$. Then $P_{t} f(e)=0$ for all $t$. This implies $\left\langle U, V_{i}\right\rangle=0$ for all $i$.

For $Y \in \mathcal{P}$, there exist $k \in K$ and $H \in \overline{\mathcal{A}_{+}}$such that $Y=A d(k) H$. If $H \in \mathcal{A}_{+}$, then such a $Y$ will be called regular. The regular elements of $\mathcal{P}$ form an open subset of $\mathcal{P}$ whose complement has a positive codimension. For $G=S L(n, R), \mathcal{P}$ is the space of symmetric matrices of trace zero, and $Y \in \mathcal{P}$ is regular if and only if $Y$ has distinct eigenvalues.

Let $Y \in \mathcal{P}$ be regular with $Y=A d(k) H$ for some $H \in \mathcal{A}_{+}$, and let $g=e^{Y}$. Then $g^{n}=k e^{n H} k^{-1}$, for $n=1,2, \ldots$, form a contracting sequence. Therefore, if $T_{\mu}$ contains some $e^{Y}$, where $Y \in \mathcal{P}$ is regular, then $T_{\mu}$ contains a contracting sequence. In the proof of Proposition 1, we saw that $\operatorname{supp}(\eta) \subset T_{\mu}$. Hence, if $\operatorname{supp}(\eta)$ contains $e^{Y}$ for some regular $Y \in \mathcal{P}$, then $T_{\mu}$ contains a contracting sequence.

In Section 3, we have seen that if the Lévy measure $\eta$ is finite, then $g_{t}$ can be obtained as a solution of the $\operatorname{SDE}(7)$ for $t<T$, where $T$ is an exponential random variable independent of the driving Wiener process $w_{t}$. By the support theorem for diffusion processes (see e.g. Theorem 8.1 in Chapter VI of [8]), $T_{\mu}$ contains the elements of the form

$$
\exp \left(\sum_{i=1}^{n} c_{i} U_{i}+c_{0} U_{0}\right)
$$

where $c_{1}, \ldots, c_{n}$ are arbitrary real numbers and $c_{0}$ is an arbitrary nonnegative number. Therefore, we have the following conclusion.

Proposition 2. $T_{\mu}$ contains a contracting sequence if either of the following two conditions holds.

(i) $\operatorname{supp}(\eta)$ contains $e^{Y}$ for some regular $Y \in \mathcal{P}$.

(ii) $\eta$ is a finite measure and there exist $X \in \mathcal{L}$ and $c \geq 0$ such that $X+c U_{0}$ is a regular element of $\mathcal{P}$.

\section{The Rate of Convergence}

Let $g_{t}$ be a Lévy process in $G$, and let $g_{t}=x_{t} \exp \left(H_{t}^{+}\right) y_{t}$ and $g_{t}=n_{t} \exp \left(H_{t}\right) k_{t}$ be respectively its Cartan and Iwasawa decompositions. Now $g_{0}$ is not assumed to be the identity $e$. The left invariance of $g_{t}$ implies that $g_{t}=g_{0} g_{t}^{e}$, where $g_{t}^{e}$ is the Lévy process starting at $e$. Hence, all the convergences stated in Theorem 1 and Corollary 1 hold also for $g_{t}$, except that $\pi\left(x_{t}\right)$ and $\pi\left(n_{t}\right)$ now converge to $g_{0} z$ instead of $z$ in $B$.

In this section we will show that the limit $\tilde{H}=\lim _{t \rightarrow \infty} H_{t} / t$ exists, and we will obtain an integral formula for $\tilde{H}$. By Lemma $1, \tilde{H}$ is also the limit of $H_{t}^{+} / t$ as $t \rightarrow \infty$.

Recall that for $X \in \mathcal{G}, X=X_{\mathcal{N}}^{I}+X_{\mathcal{A}}^{I}+X_{\mathcal{K}}^{I}$ is the decomposition $\mathcal{G}=\mathcal{N}+\mathcal{A}+\mathcal{K}$, for $g \in G, g=g_{N}^{I} g_{A}^{I} g_{K}^{I}$ is the Iwasawa decomposition $G=N A K$, and for $a \in A$, $\log a \in \mathcal{A}$ is defined by $a=e^{\log a}$.

For any $g \in G$, the decomposition $g=e^{Y} k$ with $Y \in \mathcal{P}$ and $k \in K$ is unique. We define $[g]_{\mathcal{P}}=Y$ and let $\|Y\|=\langle Y, Y\rangle^{1 / 2}$. Note that $\log g_{A}^{I}$ is not the orthogonal projection of $[g]_{\mathcal{P}}$ to $\mathcal{A}$ in general. However, we have that $\left\|\log \left[k g k^{-1}\right]_{A}^{I}\right\| \leq\left\|[g]_{\mathcal{P}}\right\|$ for $g \in G$ and $k \in K$; see Exercise B.2(iv) in Chapter VI of [6]. 
Theorem 2. Assume the hypotheses of Theorem 1 and the above notations. Let $g_{t}$ be a Lévy process in $G$ with generator (5) and a finite Lévy measure $\eta$ satisfying $\int_{G}\left\|[g]_{\mathcal{P}}\right\| \eta(d g)<\infty$. Then almost surely, as $t \rightarrow \infty, H_{t} / t$ converges to some $\tilde{H} \in \mathcal{A}_{+}$given by

$$
\begin{aligned}
\tilde{H}= & \int_{K}\left\{\frac{1}{2} \sum_{i=1}^{n}\left[\left[\operatorname{Ad}(k) U_{i}\right]_{\mathcal{K}}^{I}, \operatorname{Ad}(k) U_{i}\right]_{\mathcal{A}}^{I}+\left[\operatorname{Ad}(k) U_{0}\right]_{\mathcal{A}}^{I}\right\} \nu(d k) \\
& +\int_{K} \int_{G} \log \left[k g k^{-1}\right]_{A}^{I} \eta(d g) \nu(d k),
\end{aligned}
$$

where $\nu$ is a stationary measure on $K$.

Proof. For $g \in G$, let $H(g) \in \mathcal{A}$ be defined by the Iwasawa decomposition $g=$ $n e^{H(g)} k$. Let $g_{t}^{e}$ be the Lévy process starting at $e$. By Théorème 3.5 in [5], if $E\left[\sup _{k \in K}\left\|H\left(k g_{t}^{e}\right)\right\|\right]<\infty$, then $(1 / i) H\left(k g_{i}^{e}\right)$ converges to some non-random vector in $\mathcal{A}_{+}$as $i \rightarrow \infty$. We note that by (8), the above condition in [5] is implied by our assumption $\int\left\|[g]_{\mathcal{P}}\right\| \eta(d g)<\infty$. We need to show that $H_{t} / t$ converges as $t \rightarrow \infty$ along the real axis and the limit $\tilde{H}$ is given by (9). Our proof is independent of the above theorem in [5].

Recall that $k_{t}$ is a Markov process in $K$. Suppose the theorem is proved under the additional assumption that the distribution of $k_{0}$ is a stationary measure $\nu$ on $K$. Then for $\nu$-almost all $k \in K$, the theorem holds if $k_{0}=k$. Since $k g_{t}$ and $g_{t}$ have the same $\left(\mathcal{A}_{+}\right)$-component $H_{t}^{+}$under the Cartan decomposition for any $k \in K$, we see that the theorem holds regardless of the distribution of $k_{0}$.

Therefore, without loss of generality, we may assume that the distribution of $k_{0}$ is $\nu$. In this case, $k_{t}$ is a stationary process. By the ergodic theory, for any $f \in C(K)$, as $t \rightarrow \infty, \quad(1 / t) \int_{0}^{t} f\left(k_{s}\right) d s$ converges almost surely to some random variable $X$ and $E(X)=\int_{K} f(k) \nu(d k)$.

Let us rewrite (8) as

$$
H_{t}=H_{0}+\mathcal{M}_{t}+\int_{0}^{t} F\left(k_{s}\right) d s+\int_{0}^{t} \int_{G} J\left(k_{s-}, \sigma\right) N(d s d \sigma),
$$

where $\mathcal{M}_{t}=\int_{0}^{t} \sum_{i=1}^{n}\left[A d\left(k_{s-}\right) U_{i}\right]_{\mathcal{A}}^{I} d w_{s}^{i}$ is a martingale,

$F(k)=\frac{1}{2} \sum_{i=1}^{n}\left[\left[\operatorname{Ad}(k) U_{i}\right]_{\mathcal{K}}^{I}, \operatorname{Ad}(k) U_{i}\right]_{\mathcal{A}}^{I}+\left[\operatorname{Ad}(k) U_{0}\right]_{\mathcal{A}}^{I} \quad$ and $\quad J(k, g)=\log \left[k g k^{-1}\right]_{A}^{I}$.

We note that $\|J(k, g)\| \leq\left\|[g]_{\mathcal{P}}\right\|$.

It is easy to see that $\mathcal{M}_{t} / t \rightarrow 0$ as $t \rightarrow \infty$. By the ergodic theory mentioned above, $\int_{0}^{t} F\left(k_{s}\right) d s / t$ converges almost surely to an $\mathcal{A}$-valued random variable $H^{\prime}$ with $E\left(H^{\prime}\right)=\int_{K} F(k) \nu(d k)$.

To show the convergence of $(1 / t) \int_{0}^{t} \int_{G} J\left(k_{s-}, g\right) N(d s d g)$, let us introduce two discrete time processes, $x_{n}$ and $\bar{x}_{n}$, for $n=1,2,3, \ldots$, defined by

$$
x_{n}=\int_{n-1}^{n} \int_{G} J\left(k_{s-}, g\right) N(d s d g) \quad \text { and } \quad \bar{x}_{n}=\int_{n-1}^{n} \int_{G}\left\|J\left(k_{s-}, g\right)\right\| N(d s d g) .
$$

Both are stationary processes, since $k_{t}$ is stationary, and $\left\|x_{n}\right\| \leq \bar{x}_{n}$. Note that

$$
E\left(\bar{x}_{1}\right) \leq E \int_{0}^{1} \int_{G}\left\|[g]_{\mathcal{P}}\right\| N(d s d g)=\int_{G}\left\|[g]_{\mathcal{P}}\right\| \eta(d g)<\infty .
$$


It follows, by the law of large numbers, that $\sum_{i=1}^{n} x_{i} / n$ converges almost surely to some $\mathcal{A}$-valued random variable $H^{*}$ whose expectation is given by

$$
E\left(H^{*}\right)=E\left(x_{1}\right)=E\left\{\int_{0}^{1} \int_{G} J\left(k_{s}, g\right) \eta(d g) d s\right\}=\int_{K} \int_{G} J(k, g) \eta(d g) \nu(d k) .
$$

On the other hand, $\sum_{i=1}^{n} \bar{x}_{i} / n$ also converges; hence, $\bar{x}_{n} / n \rightarrow 0$. This implies that

$$
\sup _{n-1 \leq t \leq n} \int_{n-1}^{t}\left\|J\left(k_{s-}, g\right)\right\| N(d s d g) / n \rightarrow 0 \quad \text { as } n \rightarrow \infty .
$$

Therefore, $(1 / t) \int_{0}^{t} \int_{G} J\left(k_{s-}, g\right) N(d s d g) \rightarrow H^{*}$ as $t \rightarrow \infty$.

We have proved that as $t \rightarrow \infty, H_{t} / t$ converges almost surely to some $\mathcal{A}$-valued random variable whose expectation is equal to

$$
\tilde{H}=\int_{K} F(k) \nu(d k)+\int_{K} \int_{G} J(k, g) \eta(d g) \nu(d k) .
$$

As this random variable obviously belongs to the $\sigma$-field $\mathcal{G}_{s}=\sigma\left\{g_{s}^{-1} g_{t} ; t>s\right\}$ for any $s>0$, and the process $g_{t}$ has independent increments, by the zero-one law it must be a constant.

In [11], under the additional assumption that $\eta=0$ and the Haar measure on $K$ is a stationary measure, we derived (9) and used it to obtain an explicit expression for $\tilde{H}$.

\section{Stability of Stochastic Flows: Lyapunov Exponents}

Recall that $G=N A K$ is the Iwasawa decomposition. Let $Q$ be a closed subgroup of $G$ containing $N A$. Via the right action of $G$ on the left coset space $Q \backslash G$, any (left invariant) Lévy process $g_{t}$ with $g_{0}=e$ can be naturally regarded as a stochastic flow on $Q \backslash G$, whose one point motion $Q g g_{t}$ is a Markov process in $Q \backslash G$ starting from the point $Q g$. We will use $g_{t}$ to denote both the Lévy process in $G$ and the induced stochastic flow on $Q \backslash G$.

In general, the local stability of a stochastic flow $\phi_{t}$ on a compact manifold $S$ can be described by its Lyapunov exponents, which are defined to be the limit of $(1 / t) \log \left\|D \phi_{t}(v)\right\|$ as $t \rightarrow \infty$, for some nonzero tangent vector $v$, where $D \phi_{t}$ is the differential map of $\phi_{t}$ and $\|\cdot\|$ is a metric on $S$. Under a fairly general condition, it can be shown (see [3]) that the Lyapunov exponents $c_{1}>c_{2}>\cdots>c_{r}$ are nonrandom and, for almost all $\omega$ and $x \in S$, the associated tangent vectors form a filtration of subspaces of the tangent space $T_{x} S$ :

$$
T_{x} S=V_{1} \supset V_{2} \supset \cdots \supset V_{r} \supset V_{r+1}=\{0\}
$$

such that if $v \in V_{i}-V_{i+1}$, for $i=1,2, \ldots, r$, then $\lim _{t \rightarrow \infty}(1 / t) \log \left\|D \phi_{t}(v)\right\|=c_{i}$. The Lyapunov exponents, which are independent of the metric used above, give the exponential rates at which the length of a tangent vector grows or decays under the flow $\phi_{t}$ as $t \rightarrow \infty$.

Any point in $Q \backslash G$ can be represented by $Q k$ for some $k \in K$. Fix such a $k$. Let $k g_{t}=n_{t} a_{t} k_{t}$ be the Iwasawa decomposition with $a_{t}=\exp \left(H_{t}\right)$. By the discussion at the beginning of the last section, the convergences stated in Corollary 1 hold for the Iwasawa decompositions of $k_{t}$ if the hypotheses of Theorem 1 are satisfied. Moreover, if the integrability assumption in Theorem 2 is also satisfied, then the limit $\tilde{H}=\lim _{t \rightarrow \infty} H_{t} / t$ exists in $\mathcal{A}_{+}$. 
Any $Y \in \mathcal{G}$ can be regarded as a tangent vector of $Q \backslash G$ at the point $Q e$, the tangent vector to the curve $s \mapsto Q e^{s Y}$ at $s=0$. It is a zero vector if $Y \in \mathcal{Q}$, the Lie algebra of $Q$. Its image $D g(Y)$ under the differential map of $g \in G$ is the tangent vector to the curve $s \mapsto Q e^{s Y} g$ at $s=0$.

Because $Q \supset N A$, we have

$$
Q e^{Y} k g_{t}=Q a_{t}^{-1} n_{t}^{-1} e^{Y} n_{t} a_{t} k_{t}=Q \exp \left(A d\left(a_{t}^{-1} n_{t}^{-1}\right) Y\right) k_{t} .
$$

Hence, $D g_{t}(D k(Y))=D k_{t}\left(A d\left(a_{t}^{-1} n_{t}^{-1}\right) Y\right)$.

The tangent space of $Q \backslash G$ at $Q e$ can be identified with the orthogonal complement $\mathcal{Q}^{\prime}$ of $\mathcal{Q}$ in $\mathcal{G}$. For $X \in \mathcal{G}$, we define $\|X\|_{Q \backslash G}$ to be $\left\|X^{\prime}\right\|$, where $X^{\prime}$ is the orthogonal projection of $X$ to $\mathcal{Q}^{\prime}$.

Since $K$ is compact, $Q \backslash G$ possesses a metric invariant under the right action of $K$. Using this metric and noting that the Lyapunov exponents are independent of the metric, we see that the Lyapunov exponent of the flow $g_{t}$ corresponding to the vector $D k(Y)$ is given by

$$
\lim _{t \rightarrow \infty}(1 / t) \log \left\|A d\left(a_{t}^{-1} n_{t}^{-1}\right) Y\right\|_{Q \backslash G} .
$$

For any positive root $\alpha$, let $\mathcal{Q}_{\alpha}^{\prime}$ be the orthogonal complement of $\mathcal{G}_{\alpha} \cap \mathcal{Q}$ in $\mathcal{G}_{\alpha}$, and let $\mathcal{Q}_{0}^{\prime}$ be the orthogonal complement of $\mathcal{M} \cap \mathcal{Q}$ in $\mathcal{M}$. Then because $N A \subset Q$,

$$
\mathcal{Q}^{\prime}=\mathcal{Q}_{0}^{\prime} \oplus \sum_{\alpha>0} \mathcal{Q}_{\alpha}^{\prime}
$$

Let $X=\operatorname{Ad}\left(n_{\infty}^{-1}\right) Y$ and assume that $X$ is nonzero and is contained $\mathcal{Q}_{\alpha}^{\prime}$, where $\alpha$ may be zero. Then for large $t$,

$$
\begin{aligned}
& A d\left(a_{t}^{-1} n_{t}^{-1}\right) Y=\operatorname{Ad}\left(a_{t}^{-1}\right) \operatorname{Ad}\left(n_{t}^{-1}\right) \operatorname{Ad}\left(n_{\infty}\right) X=\operatorname{Ad}\left(a_{t}^{-1}\right) \operatorname{Ad}\left(n_{t}^{-1} n_{\infty}\right) X \\
\approx & A d\left(a_{t}^{-1}\right) X=A d\left(\exp \left(-H_{t}\right)\right) X=\exp \left(-a d\left(H_{t}\right)\right) X=\exp \left(-\alpha\left(H_{t}\right)\right) X .
\end{aligned}
$$

The last expression above behaves like $\exp (-\alpha(\tilde{H}) t) X$ because $H_{t} / t \rightarrow \tilde{H}$. Since $\|X\|_{Q \backslash G}=\|X\|$, except to justify the above $\approx$, we see that for a nonzero $Y \in$ $\operatorname{Ad}\left(n_{\infty}\right) Q_{\alpha}^{\prime}$

$$
\lim _{t \rightarrow \infty}(1 / t) \log \left\|D g_{t}(D k(Y))\right\|=\lim _{t \rightarrow \infty}(1 / t) \log \left\|A d\left(a_{t}^{-1} n_{t}^{-1}\right) Y\right\|_{Q \backslash G}=-\alpha(\tilde{H}) .
$$

Note that $\|\cdot\|$ is used above to denote the metric on $Q \backslash G$.

The $\approx$ in (11) will be justified later. We recall that $n_{t} a_{t} k_{t}$ is the Iwasawa decomposition of $k g_{t}$. To emphasize the dependence on $k$, we will write $n_{t}^{k}$ for $n_{t}=\left(k g_{t}\right)_{N}^{I}$.

Theorem 3. Let $g_{t}$ be a Lévy process in $G$ with $g_{0}=e$, considered as a stochastic flow on $Q \backslash G$ via the right action of $G$. Assume the hypotheses of Theorem 1 and 2, and the notations introduced above. Then the Lyapunov exponents $-\lambda_{1}>-\lambda_{2}>$ $\cdots>-\lambda_{r}$ are the distinct values of $-\alpha(\tilde{H})$, where $\alpha$ is any positive root or zero such that $\mathcal{Q}_{\alpha}^{\prime} \neq\{0\}$, and the associated filtration of the tangent space at the point $Q k$ is given by $T_{Q k}(Q \backslash G)=V_{1} \supset V_{2} \supset \cdots \supset V_{r}$, where $V_{i}$ is the direct sum of $\operatorname{Dk}\left(\operatorname{Ad}\left(n_{\infty}^{k}\right) \mathcal{Q}_{\alpha}^{\prime}\right)$ for $\alpha(\tilde{H}) \geq \lambda_{i}$.

We note that all the Lyapunov exponents are nonpositive, and the highest exponent is zero if and only if $\mathcal{Q}_{0}^{\prime} \neq\{0\}$. 


\section{Proof of Theorem 3}

To prove Theorem 3 completely, we need to justify the $\approx$ in $(11)$. We need to show that if $\alpha$ is a root and $X$ is a nonzero root vector of $\alpha,\left\|A d\left(a_{t}^{-1} n_{t}^{-1} n_{\infty}\right) X\right\|_{Q \backslash G}$ has exponential growth rate $-\alpha(\tilde{H})$.

Let $\alpha_{1}, \ldots, \alpha_{m}$ be the list of all roots including zero, repeated as many times as their multiplicities, and let $X_{1}, \ldots, X_{m}$ be the corresponding root vectors which span $\mathcal{G}$. We may assume that $X_{i}$ are orthogonal and each is contained either in $\mathcal{Q}$ or in $\mathcal{Q}^{\prime}$. We can write

$$
A d\left(n_{t}^{-1} n_{\infty}\right) X_{i}=\sum_{j} h_{i j}(t) X_{j}
$$

We may assume that the roots $\alpha_{i}$ are ranged in such a way that $\alpha_{1}(\tilde{H}) \geq \alpha_{2}(\tilde{H}) \geq$ $\cdots \geq \alpha_{m}(\tilde{H})$. Since $\left[\mathcal{G}_{\alpha}, \mathcal{G}_{\beta}\right] \subset \mathcal{G}_{\alpha+\beta}$, we see that for $n \in N$ and $X \in \mathcal{G}_{\alpha}, \operatorname{Ad}(n) X=$ $X+Y$ for some $Y \in \sum_{\beta \in \Theta} \mathcal{G}_{\alpha-\beta}$, where $\Theta$ is the set of nontrivial linear combinations of positive roots with nonnegative integer coefficients. It follows that $h_{i i}(t)=1$ and $h_{i j}(t)=0$ if $\alpha_{j} \neq \alpha_{i}-\beta$ for some $\beta \in \Theta$. In particular, $h_{i j}(t)=0$ if $i>j$, or if $\alpha_{i}(\tilde{H})=\alpha_{j}(\tilde{H})$ but $i \neq j$.

We have

$$
A d\left(a_{t}^{-1} n_{t}^{-1} n_{\infty}\right) X_{i}=h_{i i}(t) e^{\alpha_{i}\left(-H_{t}\right)} X_{i}+\sum_{j>i} h_{i j}(t) e^{\alpha_{j}\left(-H_{t}\right)} X_{j} .
$$

Note that the terms on the right hand side of the above are mutually orthogonal and the first term has the desired exponential growth rate $-\alpha_{i}(\tilde{H})$. In order to prove our claim, it suffices to show that the exponential growth rates of other terms are not greater. In fact, it is enough to show the following weaker statement. For any $\varepsilon>0$,

$$
\left|h_{i j}(t)\right| \leq e^{-t\left[\alpha_{i}(\tilde{H})-\alpha_{j}(\tilde{H})-\varepsilon\right]} \quad \text { for sufficiently large } t>0 .
$$

For $g \in G$, let $\tilde{g}=A d(g): \mathcal{G} \rightarrow \mathcal{G}$ and let $\|\tilde{g}\|$ be the usual operator norm defined by $\|\tilde{g}\|=\sup _{X \in \mathcal{G},\|X\|=1}\|\tilde{g} X\|$. Since the inner product $\langle\cdot, \cdot\rangle$ on $\mathcal{G}$ is $\operatorname{Ad}(K)$ invariant, $\|\tilde{k}\|=1$ for $k \in K$. Using the above $X_{i}$ as a basis of $\mathcal{G}$, we may regard $\tilde{g}$ as an $m$ by $m$ matrix. Since $G$ is unimodular, $\operatorname{det}(\tilde{g})=1$; hence, $\tilde{G}=\{\tilde{g} ; g \in G\}$ is a subgroup of $S L(m, R)$. We note that the matrix norm of $\tilde{g}$, defined to be the square root of $\sum_{i, j}\left(\tilde{g}_{i j}\right)^{2}$, in general is greater than the operator norm $\|\tilde{g}\|$; but the two norms are equivalent.

Consider the process $\tilde{g}_{t}=A d\left(g_{t}\right)$, which may be regarded as a (left invariant) Lévy process in $\tilde{G}$.

Lemma 2. For any $t>0$,

$$
E\left[\sup _{0 \leq s \leq t} \log \left\|\tilde{g}_{s}\right\|\right]<\infty .
$$

The same inequality holds also for $\tilde{g}_{t}^{-1}$.

Proof. First assume $\eta=0$. Then $\tilde{g}_{t}$ can be obtained by solving a SDE of the following form:

$$
d \tilde{g}_{t}=\sum_{i=1}^{m} \tilde{g}_{t} \tilde{X}_{i} \circ d w_{t}^{i}+\tilde{g}_{t} \tilde{X}_{0} d t
$$


where the $\tilde{X}_{i}$ are some matrices. The coefficients of such an SDE satisfy a global Lipschitz condition; hence, by the method of successive approximation of strong solutions (see, for example, the discussion after Theorem 3.1 in Chapter 4 of [8]), one see that for any $t>0$, there exists a constant $C_{t}>0$ such that

$$
E\left[\sup _{0 \leq s \leq t}\left\|\tilde{g}_{s}\right\|^{2}\right] \leq C_{t} .
$$

The same holds also for $\tilde{g}_{t}^{-1}$ because $\tilde{g}_{t}^{-1}$ satisfies an SDE similar to (16) but with $\tilde{g}_{t} \tilde{X}_{i}$ replaced by $\tilde{X}_{i} \tilde{g}_{t}^{-1}$. This implies the conclusion of Lemma 2 when $\eta=0$.

Now we consider the general case when $\eta$ is not assumed to be zero, but is finite and satisfies the integrability condition: $\int_{G}\left\|[g]_{\mathcal{P}}\right\| \eta(d g)<\infty$. Via the map $g \mapsto \tilde{g}$ from $G \rightarrow \tilde{G}, \eta$ induces a finite measure $\tilde{\eta}$ on $\tilde{G}$, which is the Lévy measure of $\tilde{g}_{t}$. Since $g=k e^{Y}$ for some $k \in K$ and $Y \in \mathcal{P}$, and $Y=[g]_{\mathcal{P}}$,

$$
\|\tilde{g}\|=\left\|A d\left(e^{Y}\right)\right\|=\left\|e^{a d(Y)}\right\| \leq e^{c\|Y\|}=\exp \left(c\left\|[g]_{\mathcal{P}}\right\|\right)
$$

for some constant $c>0$. We see that $\tilde{\eta}$ satisfies $c_{1}=\int \log \|\tilde{g}\| \tilde{\eta}(d \tilde{g})<\infty$.

Let $\lambda=\tilde{\eta}(\tilde{G})$. By Section 3, there are random times $0=T_{0}<T_{1}<T_{2}<\cdots$ with independent and exponentially distributed $T_{i}-T_{i-1}$ of mean $\lambda$ and independent $\tilde{G}$-valued random variables $\sigma_{1}, \sigma_{2}, \sigma_{3}, \ldots$ with common distribution $\tilde{\eta} / \lambda$ such that $\tilde{g}_{t}$ can be obtained by solving the SDE (16) for $T_{i}<t<T_{i+1}$ and letting it jump at $t=T_{i}$ according to $\tilde{g}_{t}=\tilde{g}_{t-} \sigma_{i}$. Let $A_{i}$ be the event that there are $i$ jumps by time $t$. We have

$$
\begin{aligned}
E\left[\sup _{0 \leq s \leq t} \log \left\|\tilde{g}_{s}\right\|\right] & =\sum_{i=0}^{\infty} E\left[\sup _{0 \leq s \leq t} \log \left\|\tilde{g}_{s}\right\| \mid A_{i}\right] P\left(A_{i}\right) \\
& =\sum_{i=0}^{\infty} E\left[\sup _{0 \leq s \leq t} \log \left\|\tilde{g}_{s}\right\| \mid A_{i}\right] e^{-\lambda t}(\lambda t)^{i} / i !
\end{aligned}
$$

On $A_{i}, \tilde{g}_{s}=\tau_{1} \sigma_{1} \tau_{2} \sigma_{2} \cdots \tau_{j} \sigma_{j} \tau^{\prime}$ for some $j \leq i$, where $\tau_{k}$ is obtained by solving (16) in $\left(T_{k-1}, T_{k}\right)$ and $\tau^{\prime}$ is obtained by solving (16) in $\left(T_{j}, s\right)$. Since $E\left[\left\|\tau_{k}\right\|^{2}\right]$ and $E\left[\left\|\tau^{\prime}\right\|^{2}\right]$ have been shown to be bounded by $C_{t}$, and $E\left[\log \left\|\sigma_{k}\right\|\right]=\int \log \|\tilde{g}\| d \tilde{\eta} / \lambda \leq$ $c_{1} / \lambda$, we have

$$
E\left[\sup _{0 \leq s \leq t} \log \left\|\tilde{g}_{s}\right\| \mid A_{i}\right]=(i+1) C_{t}+i c_{1} / \lambda .
$$

Hence, $E\left[\sup _{0 \leq s \leq t} \log \left\|\tilde{g_{s}}\right\|\right]<\infty$. Since $\left\|\left[g^{-1}\right]_{\mathcal{P}}\right\|=\left\|[g]_{\mathcal{P}}\right\|$, the above argument can be easily modified to prove the same conclusion for $\tilde{g}_{t}^{-1}$. Lemma 2 is proved.

Recall that for $g \in G, g=g_{N}^{I} g_{A}^{I} g_{K}^{I}$ is the Iwasawa decomposition $G=N A K$. For simplicity, we will omit the superscript $I$ in the sequel.

Lemma 3. For any $\varepsilon>0$, almost surely, $\sup _{k \in K}\left\|\left(k g_{t}^{-1} g_{t+1}\right) \tilde{N}\right\| \leq e^{\varepsilon t}$ for sufficiently large $t>0$.

Proof. We note that $g_{t+s}=g_{t}\left(g_{s}^{\prime} \circ \theta_{t}\right)$, where $\theta_{t}$ is the time shift and $g_{s}^{\prime}$ is an independent copy of the same Lévy process. Let $i$ be a positive integer. For $i \leq t \leq i+1, g_{t}^{-1} g_{t+1}=\left(g_{t-i}^{\prime} \circ \theta_{i}\right)^{-1} g_{i}^{-1} g_{i+1}\left(g_{t-i}^{\prime \prime} \circ \theta_{i+1}\right)$, where $g_{t}^{\prime}$ and $g_{t}^{\prime \prime}$ are two independent copies of the same Lévy process. Since $g_{i}^{-1} g_{i+1}$ is identical in law with 
$g_{1}$, by Lemma 2, we have

$$
\begin{aligned}
& E\left[\sup _{i \leq t \leq i+1} \log \left\|\left(g_{t}^{-1} g_{t+1}\right)^{\sim}\right\|\right] \\
\leq & E\left[\sup _{0 \leq s \leq 1} \log \left\|\left(g_{s}^{\prime-1}\right)^{\sim}\right\|\right]+E\left[\log \left\|\left(g_{i}^{-1} g_{i+1}\right)^{\sim}\right\|\right]+E\left[\sup _{0 \leq s \leq 1} \log \left\|\left(g_{s}^{\prime \prime}\right)^{\sim}\right\|\right]<\infty .
\end{aligned}
$$

For $g \in G$ and $k \in K, k g=(k g)_{N}(k g)_{A} k^{\prime}$ for some $k^{\prime} \in K$ and $(k g)_{N}=$ $k g k^{\prime-1}(k g)_{A}^{-1}$. For $a \in A, \tilde{a}$ is a diagonal matrix such that the product of its diagonal entries is equal to one. This implies that $\left\|\tilde{a}^{-1}\right\| \leq\|\tilde{a}\|^{m-1}$. On the other hand, from $(k g)_{N}(k g)_{A}=k g k^{\prime-1}$ and the fact that $(k g) \tilde{A}$ and $(k g) \tilde{N}(k g) \tilde{A}$ have the same diagonal, we see that $\|(k g) \tilde{A}\| \leq\|\tilde{g}\|$. Therefore,

$$
\|(k g) \tilde{N}\| \leq\|\tilde{g}\| \cdot\left\|[(k g) \tilde{A}]^{-1}\right\| \leq\|\tilde{g}\|^{m} .
$$

We have

$$
E\left[\sup _{i \leq t \leq i+1} \sup _{k \in K} \log \left\|\left(k g_{t}^{-1} g_{t+1}\right) \tilde{N}\right\|\right] \leq m E\left[\sup _{i \leq t \leq i+1} \log \left\|\left(g_{t}^{-1} g_{t+1}\right)^{\sim}\right\|\right]<\infty .
$$

For $i=1,2,3, \ldots, u_{i}=\sup _{i \leq t \leq i+1} \sup _{k \in K} \log \left\|\left(k g_{t}^{-1} g_{t+1}\right) \tilde{N}\right\|$ are iid random variables with finite expectation. As a consequence of the strong law of large numbers, $(1 / i) u_{i} \rightarrow 0$ as $i \rightarrow \infty$. This implies the conclusion of Lemma 3 .

The following lemma establishes (15) and, hence, completes the proof of Theorem 3. It has been pointed out by the anonymous referee that an inequality of similar nature for discrete times appears in Raghunathan's proof of the Oseledec ergodic theorem (Israel Journal of Math. 32, 356-362, 1979; MR 81f:60016).

Lemma 4. For $h_{i j}(t)$ defined in (13), we have, for any $\varepsilon>0$, almost surely,

$$
\left|h_{i j}(t)\right| \leq \exp \left\{-t\left[\alpha_{i}(\tilde{H})-\alpha_{j}(\tilde{H})-\varepsilon\right]\right\}
$$

for sufficiently large $t>0$.

Proof. Fix an arbitrarily small $\delta>0$. Let $g_{t}=n_{t} a_{t} k_{t}$ be the Iwasawa decomposition. Then $g_{t}^{-1} g_{t+1}=k_{t}^{-1} a_{t}^{-1} n_{t}^{-1} n_{t+1} a_{t+1} k_{t+1}$ and

$$
k_{t} g_{t}^{-1} g_{t+1}=\left(a_{t}^{-1} n_{t}^{-1} n_{t+1} a_{t}\right) a_{t}^{-1} a_{t+1} k_{t+1} .
$$

It follows that $n_{t}^{-1} n_{t+1}=a_{t}\left(k_{t} g_{t}^{-1} g_{t+1}\right)_{N} a_{t}^{-1}$. We will write $\tilde{n}(t)$ for $\left(k_{t} g_{t}^{-1} g_{t+1}\right) \tilde{N}$. Let $\tilde{n}(t) X_{i}=\sum_{j} c_{i j}(t) X_{j}$. Then

$$
A d\left(n_{t}^{-1} n_{t+1}\right) X_{i}=\tilde{a}_{t} \tilde{n}(t) \tilde{a}_{t}^{-1} X_{i}=e^{-\alpha_{i}\left(H_{t}\right)} \tilde{a}_{t} \tilde{n}(t) X_{i}=\sum_{j} e^{-\left(\alpha_{i}-\alpha_{j}\right)\left(H_{t}\right)} c_{i j}(t) X_{j} .
$$

The coefficients $c_{i j}(t)$ have properties similar to those stated for $h_{i j}(t)$ after (13). In particular, $c_{i j}(t)=0$ if $i \neq j$ and $\alpha_{j} \neq \alpha_{i}-\beta$ for some $\beta \in \Theta$.

By Lemma 3, the norm of the matrix $\left\{c_{i j}(t)\right\}$ is $\leq e^{c \delta t / 2}$ for sufficiently large $t>0$, where $c=\min \left\{\left(\alpha_{i}-\alpha_{j}\right)(\tilde{H}) ;\left(\alpha_{i}-\alpha_{j}\right)(\tilde{H})>0\right\}$. Since $H_{t} / t \rightarrow \tilde{H}$ as $t \rightarrow \infty$, we have

$$
e^{-\left(\alpha_{i}-\alpha_{j}\right)\left(H_{t}\right)} c_{i j}(t)=e^{-t(1-\delta)\left(\alpha_{i}-\alpha_{j}\right)(\tilde{H})} b_{i j}(t),
$$

where the matrix $\left\{b_{i j}(t)\right\}$ has norm $\leq 1$ for sufficiently large $t>0$. 
We have $A d\left(n_{t}^{-1} n_{\infty}\right) X_{i}=\lim _{k \rightarrow \infty} A d\left(n_{t}^{-1} n_{t+1} n_{t+1}^{-1} n_{t+2} \cdots n_{t+k}^{-1} n_{t+k+1}\right) X_{i}$ and

$$
\begin{aligned}
A d & \left(n_{t}^{-1} n_{t+1} n_{t+1}^{-1} n_{t+2} \cdots n_{t+k}^{-1} n_{t+k+1}\right) X_{i} \\
= & A d\left(n_{t}^{-1} n_{t+1}\right) A d\left(n_{t+1}^{-1} n_{t+2}\right) \cdots A d\left(n_{t+k}^{-1} n_{t+k+1}\right) X_{i} \\
= & \sum_{j_{1}, \ldots, j_{k}, j} e^{-(t+k)(1-\delta)\left(\alpha_{i}-\alpha_{j_{1}}\right)(\tilde{H})} b_{i j_{1}}(t+k) \\
& \quad \times e^{-(t+k-1)(1-\delta)\left(\alpha_{j_{1}}-\alpha_{j_{2}}\right)(\tilde{H})} b_{j_{1} j_{2}}(t+k-1) \cdots \\
& \cdots e^{-(t+1)(1-\delta)\left(\alpha_{j_{k-1}}-\alpha_{j_{k}}\right)(\tilde{H})} b_{j_{k-1} j_{k}}(t+1) e^{-t(1-\delta)\left(\alpha_{j_{k}}-\alpha_{j}\right)(\tilde{H})} b_{j_{k} j}(t) X_{j} \\
= & \sum_{j} e^{-t(1-\delta)\left(\alpha_{i}-\alpha_{j}\right)(\tilde{H})} C_{i j}(t, k) X_{j},
\end{aligned}
$$

where $C_{i j}(t, k)$ is given by

$$
\begin{gathered}
\sum_{j_{1}, \ldots, j_{k}} e^{-k(1-\delta)\left(\alpha_{i}-\alpha_{j_{1}}\right)(\tilde{H})} b_{i j_{1}}(t+k) e^{-(k-1)(1-\delta)\left(\alpha_{j_{1}}-\alpha_{j_{2}}\right)(\tilde{H})} b_{j_{1} j_{2}}(t+k-1) \cdots \\
\cdots e^{-(1-\delta)\left(\alpha_{j_{k-1}}-\alpha_{j_{k}}\right)(\tilde{H})} b_{j_{k-1} j_{k}}(t+1) b_{j_{k} j}(t) .
\end{gathered}
$$

The matrix $\left\{C_{i j}(t, k)\right\}$ has norm $\leq 1$. Since $A d\left(n_{t}^{-1} n_{\infty}\right) X_{i}=\sum_{j} h_{i j}(t) X_{j}$, the above shows that

$$
h_{i j}(t)=e^{-t(1-\delta)\left(\alpha_{i}-\alpha_{j}\right)(\tilde{H})} \lim _{k \rightarrow \infty} C_{i j}(t, k) .
$$

Lemma 4 is proved by letting $\varepsilon=\delta \max _{j>i}\left(\alpha_{i}-\alpha_{j}\right)(\tilde{H})$.

\section{Global Stability}

Let $g_{t}$ be a Lévy process in $G$ with $g_{0}=e$ satisfying the hypotheses stated in Theorems 1 and 2, and let $g_{t}=x_{t} \exp \left(H_{t}^{+}\right) y_{t}$ be the Cartan decomposition. We know that $H_{t}^{+} / t \rightarrow \tilde{H} \in \mathcal{A}_{+}$and we may assume $x_{t} \rightarrow x_{\infty}$ in $K$ as $t \rightarrow \infty$. Because of the non-uniqueness of $(x, y)$ in the Cartan decomposition $g=x \exp \left(H^{+}\right) y$, we have non-unique choices for $x_{t}$, hence, also for $x_{\infty}$. But all possible choices for $x_{\infty}$ are given by $x_{\infty} m$ for $m \in M$.

As before, let $Q$ be a closed subgroup of $G$ containing $N A$, and consider $g_{t}$ as a stochastic flow on $Q \backslash G$ via the right action. We can show that almost surely there is an open subset of $Q \backslash G$ with a positive-codimensional complement, which in fact is the image of a fixed subset of $Q \backslash G$ under a random "rotation", such that each component of this open set is shrunk to a "moving" positive codimensional limiting set exponentially by $g_{t}$ as $t \rightarrow \infty$. In the case of $Q \supset N A M_{0}$, where $M_{0}$ is the identity component of $M$, each limiting set is a single point. We note that in this case, all the Lyapunov exponents are strictly negative.

If $Q=N A M$, then $Q \backslash G$ is $\tilde{B}$, introduced in Section 2. We will first establish the result for $g_{t}$ on $\tilde{B}$, from which the result for $g_{t}$ on more general spaces can be read off.

Recall that $\tilde{\pi}: G \rightarrow \tilde{B}$ is the natural projection. By the Bruhat decomposition of $G$ discussed in Section $2, \Lambda=\tilde{\pi}\left(N A M N^{+}\right)$is a connected open subset of $\tilde{B}$ with a positive-codimensional complement. Let $o$ be the coset $(N A M)$. Then we may write $\Lambda=o N^{+}$via the right action of $G$ on $\tilde{B}$.

It is clear that the action of $a \in A$ fixes the point $o$. Since $a^{-1} n^{\prime} a \in N^{+}$for $a \in A$ and $n^{\prime} \in N^{+}$, we see that $a$ leaves $\Lambda$ invariant. Let $a=\exp \left(H_{t}^{+}\right)$, and 
$n^{\prime}=e^{Y}$ for some root vector $Y$ of a positive $\operatorname{root} \alpha$. Then

$$
o e^{Y} a=o \exp \left(A d\left(a^{-1}\right) Y\right)=o \exp \left(e^{a d\left(-H_{t}^{+}\right)} Y\right)=o \exp \left(e^{-\alpha\left(H_{t}^{+}\right)} Y\right) .
$$

Since $H_{t}^{+} / t \rightarrow \tilde{H}$, we see that the distance between $o n^{\prime} \exp \left(H_{t}^{+}\right)$and $o$ tends to zero at a negative exponential rate $-\alpha(\tilde{H})$ as $t \rightarrow \infty$.

Now let $\Lambda(\omega)=\Lambda x_{\infty}(\omega)^{-1}$, where $\omega$ is a fixed typical sample path. We have mentioned before that the choice for $x_{\infty}$ is not unique. However, two different choices for $x_{\infty}$ differ only by a factor $m \in M$ on the right; this amounts to replacing $x_{\infty}^{-1}$ above by $m x_{\infty}^{-1}$ for some $m \in M$. Since $\Lambda m=\Lambda$, we see that the definition of $\Lambda(\omega)$ is independent of the choice of $x_{\infty}$.

Any $z \in \Lambda(\omega)$ can be expressed as $z=$ on $^{\prime} x_{\infty}^{-1}$ for some $n^{\prime} \in N^{+}$. We have suppressed $\omega$ for simplicity:

$$
z g_{t}=o n^{\prime} x_{\infty}^{-1} x_{t} \exp \left(H_{t}^{+}\right) y_{t} \approx o n^{\prime} \exp \left(H_{t}^{+}\right) y_{t}
$$

for large $t>0$. The above $\approx$ can be easily justified. Since $K$ is compact, we may assume that the right action of $K$ leaves the distance on $\tilde{B}$ invariant. It follows that the distance between $z g_{t}$ and $o y_{t}$ tends to zero at a negative exponential rate $\leq-\inf _{\alpha>0} \alpha(\tilde{H})$. To summarize, we have

Theorem 4. Let $g_{t}$ be a Lévy process in $G$ satisfying the hypotheses of Theorems 1 and 2 with $g_{0}=e$, and let $g_{t}=x_{t} \exp \left(H_{t}^{+}\right) y_{t}$ be its Cartan decomposition. Consider $g_{t}$ as a stochastic flow on $\tilde{B}=(N A M) \backslash G$ via the right action of $G$ on $\tilde{B}$. The set $\Lambda=o N^{+}$is a connected open subset of $\tilde{B}$ with a positive-codimensional complement. For almost all $\omega$, all the points in $\Lambda x_{\infty}(\omega)^{-1}$ will converge to the "moving" point oyt $(\omega)$ exponentially under the flow $g_{t}$ in the sense that

$$
\forall z \in \Lambda x_{\infty}^{-1}, \quad \limsup _{t \rightarrow \infty} \frac{1}{t} \log \operatorname{dist}\left(z g_{t}, o y_{t}\right) \leq-\lambda,
$$

where $-\lambda$ is the highest Lyapunov exponent of $g_{t}$ on $\tilde{B}$.

Now consider $g_{t}$ as a stochastic flow on $\tilde{B}_{0}=\left(N A M_{0}\right) \backslash G$. This is a covering space of $(M A N) \backslash G$ with the covering map given by $p$ : $\left(M_{0} A N\right) g \mapsto(M A N) g$. Then $\Lambda_{0}=p^{-1}(\Lambda)$ is an open subset of $\left(M_{0} A N\right) \backslash G$ with a positive-codimensional complement. Let $u$ be the point in $\left(N A M_{0}\right) \backslash G$ represented by the $\operatorname{coset}\left(N A M_{0}\right)$. Then the connected components of $\Lambda_{0}$ are given by $u m N^{+}$for $m \in M$, and each of those is mapped diffeomorphically onto $\Lambda$ by $p$. Therefore, under the stochastic flow $g_{t}$, all the points in $u m N^{+} x_{\infty}^{-1}$ will converge to $u m y_{t}$ exponentially. We note that $u m N^{+}=u m^{\prime} N^{+}$if and only if $m^{\prime} \in M_{0} m$.

Now let $Q$ be a closed subgroup of $G$ containing $N A M_{0}$, let $p_{Q}$ be the natural map: $\left(M_{0} A N\right) \backslash G \rightarrow Q \backslash G$ defined by $\left(M_{0} A N\right) g \mapsto Q g$, and let $\Lambda_{Q}=p_{Q}\left(\Lambda_{0}\right)$. The connected components of $\Lambda_{Q}$ are given by $p_{Q}\left(u m N^{+}\right)$for $m \in M$. We note that even if $u m N^{+} \neq u m^{\prime} N^{+}, p_{Q}\left(u m N^{+}\right)$and $p_{Q}\left(u m^{\prime} N^{+}\right)$may still be the same.

Corollary 2. Assume $Q$ is a closed subgroup of $G$ containing $N A M_{0}$. Then $\Lambda_{Q}$ defined above is an open subset of $Q \backslash G$ with a positive-codimensional complement. Under the stochastic flow $g_{t}$, almost surely, all the points in each component of $\Lambda_{Q} x_{\infty}^{-1}$ will converge to a single "moving" point exponentially in the sense of (17), where $\Lambda$ and oyt should be replaced, respectively, by $\Lambda_{Q}$ and $p_{Q}(u m) y_{t}$, and $-\lambda$ is the highest Lyapunov exponent of $g_{t}$ on $Q \backslash G$. 
To illustrate the structures mentioned above, let $G=S L(n, R)$. Then $M_{0}=\{e\}$, and $\tilde{B}_{0}=(N A) \backslash G$ can be identified with $K=S O(n)$. The right action of $G$ on $K$ is given by $h g=k$ for $h \in K$ and $g \in G$ with Iwasawa decomposition $h g=n a k$. The row vectors of $k$ are obtained from the row vectors of the matrix product $h \cdot g$ by a Gram-Schmidt orthogonalization. For $\gamma \subset\{1,2, \ldots, n\}$ and a matrix $g \in G$, let $g[\gamma]$ be the determinant of the submatrix of $g$ formed by the rows and columns indexed by $\gamma$. We have $\Lambda_{0}=\{k \in K ; k[1,2, \ldots, i] \neq 0$ for $i=1,2, \ldots, n-1\}$. Each

connected component of $\Lambda_{0}$ consists of $k \in K$ with fixed signs for $k[1,2, \ldots, i]$ for all $i$. In particular, $u N^{+}=\{k \in K ; k[1,2, \ldots, i]>0$ for all $i\}$.

Remark 2. We now consider the general case of the stochastic flow $g_{t}$ on $Q \backslash G$ with $Q \supset N A$. If $Q \not \supset M$, there exists a zero Lyapunov exponent and we cannot expect the clustering around single points. However, our proof shows that there is an open subset of $Q \backslash G$ with a positive-codimensional complement such that each of its connected component is shrunk to a lower dimensional "moving" set exponentially by $g_{t}$.

For example, in the case of $Q=N A$, let $p^{\prime}: G \rightarrow(N A) \backslash G$ be the natural projection and let $\Lambda^{\prime}=p^{\prime}\left(M N^{+}\right)$. Then $\Lambda^{\prime}$ is an open subset of $(N A) \backslash G$ with a positive-codimensional complement. For almost all $\omega, g_{t}$ shrinks $\Lambda^{\prime} x_{\infty}(\omega)^{-1}$ exponentially into the lower-dimensional "moving" set $p^{\prime}(M) y_{t}$ as $t \rightarrow \infty$ in the sense that the distance between any point in $\Lambda^{\prime} x_{\infty}(\omega)^{-1}$ and the set $p^{\prime}(M) y_{t}$ tends to zero at an exponential rate $\leq-\lambda$, where $-\lambda$ is the highest negative Lyapunov exponent of $g_{t}$ on $(N A) \backslash G$.

\section{Some Stochastic Flows on Spheres}

In this section, we will consider some examples of stochastic flows on the $(n-1)$ dimensional sphere $S^{n-1}$, embedded as the unit sphere in $R^{n}$.

We will first make some general remarks about continuous Lévy processes in $G$ and the induced stochastic flow on a manifold $S$ via the right action of $G$ on $S$. Such a process can be obtained as the solution of the following SDE on $G$ :

$$
d g_{t}=\sum_{i=1}^{n} g_{t} U_{i} \circ d w_{t}^{i}+g_{t} U_{0} d t
$$

where $U_{0}, U_{1}, \ldots, U_{n} \in \mathcal{G}$ and $w_{t}=\left\{w_{t}^{i}\right\}$ is an $n$-dimensional Wiener process. By Propositions 1 and 2, the hypotheses of Theorem 1 are satisfied if $U_{1}, \ldots, U_{n}$ generate $\mathcal{G}$ and their linear span contains a regular element of $\mathcal{P}$. We note that the integrability assumption in Theorem 2 is always satisfied for a continuous Lévy process.

Any $U \in \mathcal{G}$ induces a vector field $U^{*}$ on $S$ defined by $U^{*} f(x)=\left.(d / d s) f\left(x e^{s U}\right)\right|_{s=0}$ for smooth functions $f$ on $S$ and $x \in S$. The stochastic flow $g_{t}$ on $S$ is the solution flow of the following SDE on $S$ :

$$
d x_{t}=\sum_{i=1}^{m} U_{i}^{*}\left(x_{t}\right) \circ d w_{t}^{i}+U_{0}^{*}\left(x_{t}\right) d t .
$$

Stochastic Flows in $S L(n, R)$. Let $G=S L(n, R)$. Consider its right action on $S^{n-1}$ defined by $x \mapsto x g=(x \cdot g) /\|x \cdot g\|$ for $x=\left(x_{1}, x_{2}, \ldots, x_{n}\right) \in S^{n-1}$ and $g \in G$. Here $x$ is considered as a row vector, the dot $\cdot$ denotes the usual matrix multiplication, and $\|\cdot\|$ is the Euclidean norm on $R^{n}$. Thus, any Lévy process $g_{t}$ 
in $G$ can be regarded as a stochastic flow on $S^{n-1}$ via this right action. We will assume that $g_{t}$ satisfies the hypotheses of Theorems 1 and 2 .

Let $v=(1,0, \cdots, 0)$ and $Q=\{g \in G ; v g=v\}$. Then $S^{n-1}=Q \backslash G$. It is easy to see that $Q$ consists of all matrices in $G$ whose first rows have the form $(a, 0, \cdots, 0)$ for some $a>0$. The root structure of $G=S L(n, R)$ has been described in Section 2. We note that $M$ is a discrete group, so $\mathcal{M}=\{0\}$. By Theorem 3, the stochastic flow has $n-1$ negative Lyapunov exponents: $-\lambda_{1}>-\lambda_{2}>\cdots>-\lambda_{n-1}$. Furthermore, using the right action of $N^{+}$on $S^{n-1}$, we can see that the set $\Lambda_{Q}$ in Corollary 2 has two components: $S_{1}=\left\{x=\left(x_{1}, \ldots, x_{n}\right) \in S ; x_{1}>0\right\}$ and $S_{2}=\left\{x=\left(x_{1}, \ldots, x_{n}\right) \in S ; x_{1}<0\right\}$; and the points $p_{Q}(u m)$ for $S_{1}$ and $S_{2}$ are, respectively, $v$ and $-v$. We can draw the following conclusions: For almost all $\omega$, there is a great hypercircle $C_{\omega}$ on $S^{n-1}$ such that for any two points $z_{1}$ and $z_{2}$,

(i) if $z_{1}$ and $z_{2}$ lie on the same side of $C_{\omega}$, then

$$
\limsup _{t \rightarrow \infty}(1 / t) \log \operatorname{dist}\left(z_{1} g_{t}, z_{2} g_{t}\right) \leq-\lambda_{1}
$$

(ii) if $z_{1}$ and $z_{2}$ lie on the different sides of $C_{\omega}$, then $\operatorname{dist}\left(z_{1} g_{t}, z_{2} g_{t}\right) \rightarrow 1$ (diameter of $\left.S^{n-1}\right)$ as $t \rightarrow \infty$, where dist is the distance on $S^{n-1}$ induced by the Euclidean distance.

We note that the above conclusions hold for any stochastic flow on $S^{n-1}$ as long as it is induced by a Lévy process in $S L(n, R)$ which satisfies the hypotheses of Theorems 1 and 2 .

Sometimes it is more convenient to work with a Lévy process $g_{t}$ in $G L(n, R)$, the group of $n$ by $n$ nonsingular real matrices, rather than a process in its subgroup $S L(n, R)$. The action of $G L(n, R)$ on $S^{n-1}$ is defined exactly as for $S L(n, R)$. Although $G L(n, R)$ is not semisimple, it is a direct product of $R_{+}$(the multiplication group of positive reals) and $S L(n, R)$, via the group isomorphism $g \mapsto$ $\left(\operatorname{det}(g), \operatorname{det}(g)^{-1 / n} g\right)$ from $G L(n, R)$ onto $R_{+} \times S L(n, R)$. As the action of $R_{+}$on $S^{n-1}$ is trivial, we may assume that $g_{t}$ is a Lévy process in $S L(n, R)$; hence, the above conclusions hold.

We now consider such an example. Let $E_{i j}$ be the $n$ by $n$ matrix which has 1 at place $(i, j)$ and 0 elsewhere. Such matrices form a basis of the Lie algebra of $G L(n, R)$. Consider the following $\mathrm{SDE}$ on $G L(n, R)$ :

$$
d g_{t}=\sum_{i, j=1}^{n} c_{i j} g_{t} E_{i j} \circ d w_{t}^{i j},
$$

where $w_{t}=\left\{w_{t}^{i j}\right\}$ is an $n^{2}$-dimensional Wiener process and the $c_{i j}$ are constants. The solution $g_{t}$ of this SDE with $g_{0}=I_{n}$ (the $n$ by $n$ identity matrix) is a continuous Lévy process in $G L(n, R)$. The hypotheses are satisfied if all the $c_{i j}$ are nonzero.

For $x=\left(x_{1}, \ldots, x_{n}\right) \in S^{n-1}$,

$$
x \cdot \exp \left(s E_{i j}\right)=x \cdot\left(I+s E_{i j}\right)+O\left(s^{2}\right)=\left(x_{1}, \ldots, x_{j}+s x_{i}, \ldots, x_{n}\right)+O\left(s^{2}\right)
$$

and its norm $=1+s x_{i} x_{j}+O\left(s^{2}\right)$. From this, we can show that the induced vector field $E_{i j}^{*}$ on $S^{n-1}$ is given by

$$
E_{i j}^{*}(x)=x_{i}\left(\partial / \partial x_{j}\right)-x_{i} x_{j} \nabla
$$

where $\nabla=\sum_{i=1}^{n} x_{i}\left(\partial / \partial x_{i}\right)$. This is the orthogonal projection to $S^{n-1}$ of the vector field $x_{i}\left(\partial / \partial x_{j}\right)$ on $R^{n}$. The stochastic flow $g_{t}$ is the solution flow of the following 
SDE on $S^{n-1}$ :

$$
d x_{t}=\sum_{i, j=1}^{n} c_{i j} E_{i j}^{*}\left(x_{t}\right) \circ d w_{t}^{i j} .
$$

If all $c_{i j}=1$, then the one point motion of the stochastic flow is Brownian motion in $S^{n-1}$ (see [10]).

Stochastic Flows in the Lorentz Group. The Lorentz group on $R^{n+1}$ is the group of linear transformations, or $(n+1)$ by $(n+1)$ real matrices, which leave the quadratic form $x_{0}^{2}-\sum_{i=1}^{n} x_{i}^{2}$ invariant. We will use $x_{0}, x_{1}, \ldots, x_{n}$ as Cartesian coordiates on $R^{n+1}$, and $x=\left(x_{1}, \ldots, x_{n}\right)$ as coordinates on the subspace $R^{n}$ which contains $S^{n-1}$ as the unit sphere. The Lorentz group has four connected components. Let $G=S O_{+}(1, n)$ be the identity component.

We now define a right action of $G$ on $S^{n-1}$. For $x \in S^{n-1}$ and $g \in G$, let $(v, y)=(1, x) \cdot g$, where $v$ is a real number, $y \in R^{n}$ and the dot $\cdot$ denotes the matrix multiplication. Then $v^{2}-\|y\|^{2}=1-\|x\|^{2}=0$, and hence, $y / v \in S^{n-1}$. We define $x g=y / v$. This is a well defined right action of $G$ on $S^{n-1}$. Via this action, any Lévy process in $G=S O_{+}(1, n)$ can be regarded as a stochastic flow on $S^{n-1}$.

The Lie algebra $\mathcal{G}$ of $G$ is the space of the matrices having the following block form:

$$
\left[\begin{array}{ll}
0 & y \\
y^{*} & B
\end{array}\right]
$$

where $B \in o(n)$ (the space of $n$ by $n$ skew-symmetric matrices), $y=\left(y_{1}, \ldots, y_{n}\right)$ is a row vector and $y^{*}$ is the transpose of $y$. For $\theta \in S^{n-1}$, let $X_{\theta}$ be such a matrix with $B=0$ and $y=\theta$. We may take $K$ to be the compact subgroup of matrices

$$
\left[\begin{array}{ll}
1 & 0 \\
0 & B
\end{array}\right]
$$

with $B \in S O(n)$. Then $\mathcal{P}$ is spanned by $X_{\theta}, \theta \in S^{n-1}$. All the maximal abelian subspaces of $\mathcal{P}$ are one dimensional. Fix a $\theta \in S^{n-1}$. We may take $\mathcal{A}$ to be such a subspace spanned by $X_{\theta}$, and $\mathcal{A}_{+}$to be the half line $\left\{c X_{\theta} ; c>0\right\}$. There are only two roots $\pm \alpha$, given by $\alpha\left(X_{\theta}\right)=1$.

Let $Q=\{g \in G ; \theta g=\theta\}$. Then $S^{n-1}=Q \backslash G$. We can show that $M$ is the group of orthogonal transformations on $R^{n}$ which fix the point $\theta$ and $Q=N A M$. We may take $\theta$ to be the point $o$ in Section 8. Since $\Lambda=o N^{+}$is a simply connected open subset of $S^{n-1}$ with a positive-codimensional complement and it is invariant under the action of $M$, the complement of $\Lambda$ has to be the point antipodal to $\theta$. Therefore, by Theorems 3 and 4 , for any stochastic flow $g_{t}$ on $S^{n-1}$ induced by a Lévy process in $S O_{+}(1, n)$ which satisfies the hypotheses of Theorems 1 and 2 , there is only one negative exponent $-\lambda$ and, for almost all $\omega$, there is a random point $x(\omega)$ on $S^{n-1}$ such that its complement is shrunk by the stochastic flow exponentially in the sense of (18), where $z_{1}$ and $z_{2}$ are any two points on $S^{n-1}$ distinct from $x(\omega)$, and $-\lambda_{1}=-\lambda$.

Let $X_{i}$ be $X_{\theta}$, where $\theta=\theta_{i}$ is the vector having 1 in the $i$-th place and zero elsewhere. Consider the following SDE on $S_{+}(1, n)$ :

$$
d g_{t}=\sum_{i=1}^{n} c_{i} g_{t} X_{i} \circ d w_{t}^{i}
$$


Its solution is a Lévy process satisfying the hypotheses if all constants $c_{i}$ are nonzero. Since $(1, x) \cdot \exp \left(s X_{i}\right)=(1, x) \cdot\left(I_{n+1}+s X_{i}\right)+O\left(s^{2}\right)=\left(1+s x_{i}, x+s \theta_{i}\right)+O\left(s^{2}\right)$, one can show that the induced vector field $X_{i}^{*}$ on $S^{n-1}$ is given by $X_{i}^{*}(x)=\left(\partial / \partial x_{i}\right)-$ $x_{i} \nabla$, which is the orthogonal projection to $S^{n-1}$ of the coordinate vector field $\left(\partial / \partial x_{i}\right)$ on $R^{n}$. Hence, $g_{t}$ is the stochastic flow mentioned at the beginning of the paper.

Finally, we will look at a simple example of a discontinuous stochastic flow. First note that the SDE $d g_{t}=g_{t} X_{1} \circ d w_{t}$, where $w_{t}$ is a one dimensional Wiener process, defines a Lévy process $g_{t}$ in $G=S O_{+}(1, n)$. It is the solution flow of the SDE $d x_{t}=X_{1}^{*}\left(x_{t}\right) \circ d w_{t}$ on $S^{n-1}$ and does not satisfy the hypotheses of Theorem 1. For $x \in S^{n-1}$, let $\phi$ be the angle between $x$ and $\theta=(1,0, \ldots, 0)$. Then the above SDE on $S^{n-1}$ can be written as

$$
d \phi_{t}=\sin \left(\phi_{t}\right) \circ d w_{t} .
$$

The solution is given by $\phi_{t}=F^{-1}\left(F\left(\phi_{0}\right) e^{-W_{t}}\right)$, where $F(\phi)=\log (\csc \phi+\cot \phi)$. We see that the stochastic flow $g_{t}$ is recurrent. In particular, under the flow, the distance between any two points will return to the original value infinitely often.

Now we add random rotations to $g_{t}$ at exponentially spaced random times. Let $\eta$ be the distribution of each random rotation and assume that $\eta$ is finite, $\eta(\{e\})=0$ and $\operatorname{supp}(\eta)=K=S O(n)$. By the discussion in Section 3, the resulting Lévy process in $G$ has generator

$$
L f(g)=(1 / 2) X_{1} X_{1} f(g)+\int_{K}[f(g \tau)-f(g)] \eta(d \tau) .
$$

This process satisfies the hypotheses of Theorems 1 and 2 . Therefore, as a stochastic flow on $S^{n-1}$, it will shrink the complement of a random point exponentially to a single point. This is a little surprising - as rotations leave distance invariant, it would have seemed that after rotations the distance between points should still remain recurrent.

\section{REFERENCES}

1. Applebaum, D. and Kunita, H., "Lévy flows on manifolds and Lévy processes on Lie groups", J. Math. Kyoto Univ. 33-4, pp 1105-1125 (1993) MR 95d:58140

2. Baxendale, P.H., "Asymptotic behaviour of stochastic flows of diffeomorphisms: two case studies", Probab. Th. Rel. Fields 73, pp 51-85 (1986) MR 88c:58073

3. Carverhill, A.P., "Flows of stochastic dynamical systems: ergodic theory", Stochastics 14, pp 273-317 (1985) MR 87c:58059

4. Elworthy, K.D., "Geometric aspects of diffusions on manifolds", (Ecole d'Eté de Probabilités de Saint Flour XVII, July 1987), Lect Notes in Math 1362, pp 276-425 (1989) MR 90c:58187

5. Guivarc'h, Y. et Raugi, A., "Frontière de Furstenberg, propriétés de contraction et convergence", Z. Wahr verw Gebiete 68, pp 187-242 (1985) MR 86h:60126

6. Helgason, S., "Differential geometry, Lie groups, and symmetric spaces", Academic Press (1978) MR 80k:53081

7. Hunt, G.A., "Semigroup of measures on Lie groups", Transactions AMS 81 (2), pp 264-293 (1956) MR 18:54a

8. Ikeda, N. and Watanabe, S., "Stochastic differential equations and diffusion processes", Second ed, North-Holland (1989) MR 90m:60069

9. Liao, M., "Stochastic flows on the boundaries of Lie groups", Stochastics and Stochastic Reports, vol 39, pp 213-237 (1992) MR 95d:58143

10. Liao, M., "Stochastic flows on the boundaries of SL(n,R)", Probab Theo \& Rel Fields 96, pp 261-281 (1993) MR 95d:58144 
11. Liao, M., "Invariant diffusion processes in Lie groups and stochastic flows", Proceedings of 1993 Summer Research Institute on Stochastic Analysis, July 1993, Cornell Univ (ed M. Cranston \& M. Pinsky), Proc. Sympos. Pure Math., vol. 57, Amer. Math. Soc., Providence, RI, 1995, pp. 575-591. MR 96d:58154

12. Malliavin, M.P. \& Malliavin, P., "Factorisations et lois limites de la diffusion horizontale audessus d'un espace Riemannien symmetrique", Lecture Notes in Math. 404, Springer-Verlag, pp 164-217 (1974) MR 50:11478

Department of Mathematics, Auburn University, Auburn, Alabama 36849

E-mail address: liaomin@mail.auburn.edu 